\title{
Oligocene-Miocene middle crustal flow in southern Tibet: geochronology of Mabja Dome
}

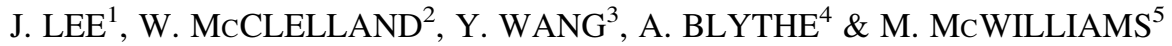 \\ ${ }^{1}$ Department of Geological Sciences, Central Washington University, Ellensburg, \\ Washington 98926, USA (e-mail: jeff@geology.cwu.edu) \\ ${ }^{2}$ Department of Geological Sciences, University of Idaho, Moscow, Idaho 83844, USA \\ ${ }^{3}$ Department of Geology, China University of Geosciences, Beijing 100083, China \\ ${ }^{4}$ Department of Earth Sciences, University of Southern California, Los Angeles, \\ California 90089, USA \\ ${ }^{5}$ Department of Geological \& Environmental Sciences, Stanford University, \\ Stanford, California 94305, USA
}

\begin{abstract}
New $\mathrm{U}-\mathrm{Pb}$ zircon, monazite, ${ }^{40} \mathrm{Ar} /{ }^{39} \mathrm{Ar}$, and apatite fission track ages provide constraints on the timing of formation and exhumation of the Mabja Dome, southern Tibet, shed light on how this gneiss dome formed, and provide important clues on the tectonic evolution of middle crustal rocks in southern Tibet. Zircons from a deformed leucocratic dyke swarm yield a $\mathrm{U}-\mathrm{Pb}$ age of $23.1 \pm 0.8 \mathrm{Ma}$, providing the first age constraint on the timing of middle crustal ductile horizontal extension in the North Himalayan gneiss domes. Zircons and monazite from a post-tectonic two-mica granite yield ages of $14.2 \pm 0.2 \mathrm{Ma}$ and $14.5 \pm 0.1$, respectively, indicating that vertical thinning and subhorizontal stretching had ceased by the middle Miocene. Mica ${ }^{40} \mathrm{Ar} /{ }^{39} \mathrm{Ar}$ ages from schists and orthogneisses increase structurally down-section from $12.85 \pm 0.13 \mathrm{Ma}$ to $17.0 \pm 0.19 \mathrm{Ma}$ and then decrease at the deepest structural levels to $13.29 \pm 0.09 \mathrm{Ma}$. Micas from the leucocratic dyke swarm and post-tectonic two-mica granites yield similar ${ }^{40} \mathrm{Ar} /{ }^{39} \mathrm{Ar}$ cooling ages of $13.48 \pm 0.13$ to $12.84 \pm 0.08 \mathrm{Ma}$. The low-temperature steps of potassium feldspar ${ }^{40} \mathrm{Ar} /{ }^{39} \mathrm{Ar}$ spectra yield ages of $c .11 .0-12.5 \mathrm{Ma}$ and apatite fission track analyses indicate the dome uniformly cooled below $c .115^{\circ} \mathrm{C}$ at $9.5 \pm 0.6 \mathrm{Ma}$. Based on these data, calculated average cooling rates across the dome range from $c .40-60^{\circ} \mathrm{C} / \mathrm{million}$ years in schist and orthogneiss and following emplacement of the leucocratic dyke swarm, to c. $350^{\circ} \mathrm{C} /$ million years following emplacement of the two-mica granites. The mylonitic foliation, peak metamorphic isograds, and mica ${ }^{40} \mathrm{Ar} /{ }^{39} \mathrm{Ar}$ chrontours are domed, whereas the low-temperature step potassium feldspar ${ }^{40} \mathrm{Ar} /{ }^{39} \mathrm{Ar}$ and apatite fission track chrontours are not, suggesting that doming occurred between 13.0 and $12.5 \mathrm{Ma}$ and at temperatures between 370 and $200^{\circ} \mathrm{C}$. Our new ages, along with field, structural and metamorphic data, indicate that the domal geometry observed at Mabja developed by middle-Miocene southward-directed thrust faulting upward and southward along a north-dipping ramp above cold Tethyan sediments. The structural, metamorphic and geochronologic histories documented at Mabja Dome are similar to those of Kangmar Dome, suggesting a common mode of occurrence of these events throughout southern Tibet. Vertical thinning and horizontal stretching, metamorphism, generation of migmatites, and emplacement of leucogranites in the domes of southern Tibet are synchronous with similar events in the Greater Himalayan Sequence that underlie the high Himalaya. These relations are consistent with previously proposed models for a ductile middle-crustal channel bounded above by the South Tibetan detachment system and below by the Main Central thrust in the High Himalaya that extended northward beneath southern Tibet.
\end{abstract}

The North Himalayan gneiss domes lie within the Tethys Himalaya south of the Indus-Tsangpo Suture Zone (ITSZ) and north of the South Tibetan detachment system (STDS) and crop out within the axis of the North Himalayan antiform (Fig. 1). These domes expose middle crustal metasedimentary rocks and orthogneisses that preserve contractional structures overprinted by moderate temperature/pressure metamorphism, high strain structures developed during vertical thinning and horizontal stretching, partial melting, and emplacement of syn- and post-tectonic leucogranites (Burg et al. 1984; Chen et al. 1990; Lee et al. 2000, 2004; Aoya et al. 2005, 2006). While the structural, metamorphic and intrusive histories in these domes are well documented, their timing is not well known. 


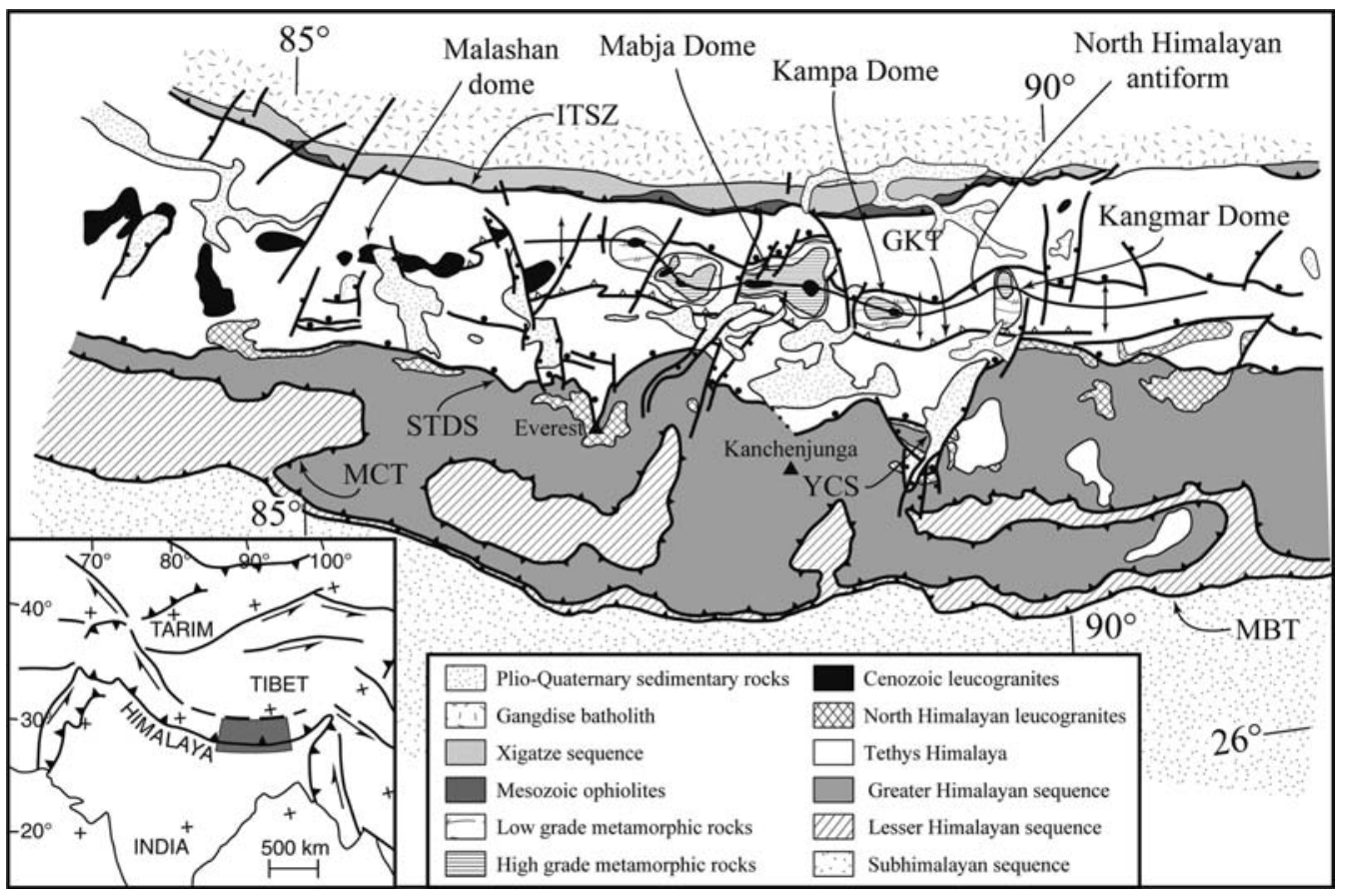

Fig. 1. Regional tectonic map of the central Himalaya orogen after Burchfiel et al. (1992) and Burg et al. (1984) showing location of the Mabja, Kangmar, Kampa and Malashan domes. GKT, Gyirong-Kangmar thrust fault system; ITSZ, Indus-Tsangpo suture zone; MBT, Main Boundary Thrust; MCT, Main Central Thrust; STDS, Southern Tibetan detachment system; YCS; Yadong cross-structure. Thrust faults represented by teeth on the hanging wall; normal faults by solid circle on the hanging wall. Inset (modified from Burchfiel et al. 1992; Tapponnier et al. 1982) shows location of regional tectonic map.

At Kangmar Dome (Fig. 1), mica ${ }^{40} \mathrm{Ar} /{ }^{39} \mathrm{Ar}$ ages showed that these rocks cooled below c. 370$335^{\circ} \mathrm{C}$ between $c$. 15 and $11 \mathrm{Ma}$, suggesting that D2 mylonitic vertical thinning and horizontal stretching deformation ended by middle Miocene (Lee et al. 2000). Apatite fission track ages indicated cooling below $c .115^{\circ} \mathrm{C}$ at $c .5 .5 \mathrm{Ma}$, implying that these rocks were exhumed to the shallow crust by late Miocene (Lee et al. 2000). At Mabja Dome (Fig. 1), slightly deformed two-mica granites exposed west of the core of the dome (Maluski et al. 1988) yielded monazite $\mathrm{U}-\mathrm{Pb}$ ages of $9.2 \pm 0.9 \mathrm{Ma}$ and $9.8 \pm 0.7 \mathrm{Ma}$ (Scharer et al. 1986) and biotite and muscovite collected from these granitic rocks and orthogneisses yielded disturbed ${ }^{40} \mathrm{Ar} /{ }^{39} \mathrm{Ar}$ spectra with total gas ages of 6-8 Ma (Maluski et al. 1988). The exact locations of these samples were not reported. More recently, $\mathrm{U}-\mathrm{Pb}$ analyses of zircon, xenotime and monazite from granite intrusions to the north of Mabja and within Mabja yielded ages of $27.5 \pm 0.5 \mathrm{Ma}$ and $14.4 \pm 0.1 \mathrm{Ma}$, respectively (Zhang et al. 2004).

To the south in the high Himalaya, the Greater Himalayan Sequence also exposes middle crust including strongly deformed, moderate-temperature/ pressure metasedimentary, orthogneissic and magmatic rocks, and both deformed and undeformed leucogranites (e.g. Le Fort et al. 1987; Hodges et al. 1988; Hubbard 1989; Burchfiel et al. 1992; Grujic et al. 1996, 2002; Murphy \& Harrison 1999; Searle 1999a, b; Walker et al. 1999; Stephenson et al. 2001; Searle et al. 2003). These rocks preserve contractional structures overprinted by mylonitic fabrics and they are bounded by two major, northdipping high-strain shear zones, the STDS normal fault at the top and the Main Central Thrust (MCT) fault at the base. In the high Himalaya, U-Pb, U$\mathrm{Th}-\mathrm{Pb}$, and ${ }^{40} \mathrm{Ar} /{ }^{39} \mathrm{Ar}$ ages indicate three major events: (1) late Eocene to late Oligocene contraction-related burial and thermal re-equilibration (e.g. Vance \& Harris 1999; Walker et al. 1999; Simpson et al. 2000); (2) early Oligocene to middle Miocene emplacement of multiple generations of both deformed and undeformed leucogranites (e.g. Noble \& Searle 1995; Hodges et al. 1996, 1998; Edwards \& Harrison 1997; Searle et al. 1997b; Wu et al. 1998; Harrison et al. 1999; Murphy \& Harrison 1999; Searle 1999a,b; Walker et al. 1999; 
Simpson et al. 2000; Daniel et al. 2003); and (3) an early to middle Miocene end to mylonitic deformation (e.g. Searle \& Rex 1989; Hodges et al. 1992; Searle et al. 1992; Walker et al. 1999; Stephenson et al. 2001). In addition, the MCT and STDS shear zones were broadly active simultaneously during the early to middle Miocene (e.g. Hubbard \& Harrison 1989; Hodges et al. 1992, 1996; Murphy \& Harrison 1999; Walker et al. 1999; Simpson et al. 2000; Stephenson et al. 2001; Daniel et al. 2003; Searle et al. 2003).

Using structural, metamorphic, geochronologic and geophysical data, and thermal-mechanical models, workers have proposed that southward extrusion and erosion of ductile middle-crust bounded above by the STDS and below by the MCT can explain the exposure of the Greater Himalayan sequence in the high Himalaya (e.g. Grujic et al. 1996, 2002; Nelson et al. 1996; Searle 1999a, b; Beaumont et al. 2001; Hodges et al. 2001; Vannay \& Grasemann 2001; Searle et al. 2003).

The parallel structural, metamorphic and intrusive histories of middle crustal rocks exposed within the Greater Himalayan Sequence, and within the North Himalayan gneiss domes, implies that these rocks represented a continuous section of middle crust beneath the high Himalaya and southern Tibet during the Oligocene and Miocene. To test this inference, geochronologic constraints on the timing of structural, metamorphic and intrusive events in the North Himalayan gneiss domes are needed. This paper presents new zircon and monazite $\mathrm{U}-\mathrm{Pb}$, mica ${ }^{40} \mathrm{Ar} /{ }^{39} \mathrm{Ar}$, and apatite fission track ages on structural, metamorphic and intrusive events at Mabja Dome, southern Tibet. Our new age data bracket the formation and exhumation of Mabja Dome, shed light on the mechanism by which this gneiss dome formed, and provide important clues on the tectonic evolution of middle crust rocks in southern Tibet.

\section{Regional setting of the North Himalayan gneiss domes}

The North Himalayan gneiss domes are exposed within the Tethyan Himalaya, approximately halfway between the ITSZ to the north and the STDS to the south (Fig. 1). The region is underlain by a Cambrian to Eocene miogeosynclinal sedimentary sequence deposited on the passive northern margin of the Indian continent (e.g. Gansser 1964; Le Fort 1975; Gaetani \& Garzanti 1991). The Tethyan Himalaya is structurally complex, exhibiting Cretaceous to Quaternary reverse faults and folds (e.g. Le Fort 1975; Searle 1983; Burg \& Chen 1984; Ratschbacher et al. 1994; Yin et al.
1994, 1999; Quidelleur et al. 1997; Searle et al. 1997a; Godin et al. 1999) and extensional structures (e.g. Molnar \& Tapponnier 1975; Armijo et al. 1986; Mercier et al. 1987; Burchfiel et al. 1992; Ratschbacher et al. 1994) in a variety of orientations.

The North Himalayan gneiss domes consist of a core of metasedimentary rocks, gneisses and granitic rocks overlain by a mantle of sedimentary and low-grade metasedimentary rocks (e.g. Burg et al. 1984; Chen et al. 1990; Lee et al. 2000, 2004; Aoya et al. 2005, 2006). These rocks preserve evidence for a north-south contractional deformation event upon which a vertical thinning and horizontal stretching deformational event was superimposed during moderate temperature/pressure metamorphism, and intrusion of leucogranites. Along-strike, the exposure of the North Himalayan gneiss domes defines the North Himalayan antiform; the domes lie in the hanging wall of the north-dipping Gyirong-Kangmar thrust fault system (GKT) (Fig. 1).

\section{Geology of Mabja Dome}

\section{Rock units}

The Mabja Dome is a migmatitic orthogneiss mantled by Palaeozoic orthogneiss and metasedimentary rocks, that in turn are overlain by Triassic and Jurassic metasedimentary and sedimentary rocks (Figs $2 \& 3$ ). The grade of metamorphism ranges from sillimanite zone at the base to unmetamorphosed at the top (Lee et al. 2004). At the base of the section is a K-feldspar augen + biotite + plagioclase + quartz \pm muscovite \pm sillimanite \pm garnet-bearing granitic migmatitic orthogneiss (og) that contains pockets and segregation banding of leucosomes and melanosomes suggesting partial melting. Structurally overlying unit og is a moderately well-exposed Palaeozoic orthogneiss and paragneiss complex (Pop) composed of dominantly $\mathrm{K}$-feldspar granitic augen gneiss with numerous pendants of metasedimentary pelite (Figs $2 \& 3$ ). The metapelites include quartzite and coarsegrained, porphyroblastic schist, which range in metamorphic grade from garnet zone at the top, through kyanite- and staurolite zones in the middle, to sillimanite zone at the base. Structurally above Pop is a sequence of Palaeozoic schist, quartzite and marble comprising units Pls, Pm, Pus and Pq (Figs $2 \& 3$ ). Overlying unit $\mathrm{Pq}$ is an aerially extensive siliciclastic Triassic sedimentary sequence (Ts) which in turn is overlain by mudstones, sandstones and limestones of Jurassic age.

The orthogneiss and metasedimentary rocks were intruded by deformed amphibolite dykes, a variably 


\section{SYMBOLS}

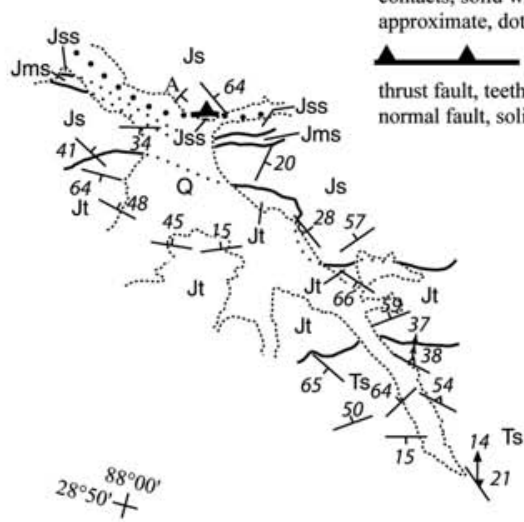

\section{EXPLANATION}

Q Quaternary deposits, undifferentiated

Mkg Miocene Kouwu two-mica granite

Miocene pegmatite dike swarm

Mdg Miocene(?) Donggong two-mica granite

Jss Jurassic sandstone

Jms Jurassic mudstone and sandstone

Js Jurassic siliciclastic rocks

$\mathrm{Jt}$ Jurassice turbidites

Ts Triassic siliciclastic rocks

$\mathrm{Pq}$ Paleozoic quartzite and marble

Pus Paleozoic upper schist

$\mathrm{Pm}$ Paleozoic marble

Pls Paleozoic lower schist

Pop Paleozoic orthogneiss, schist, and quartzite

og Basal orthogneiss

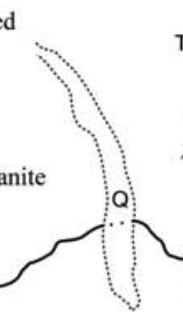

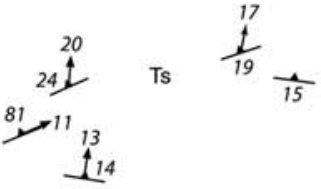

ATTITUDES

$\gamma^{10} \quad \downarrow^{77} \quad{ }^{33} y p^{44} \gtrless^{25}$

bedding; inclined, metamorphic foliations; $\mathrm{S} 1$, open overturned triangle; $\mathrm{S}$, solid triangle; $\mathrm{S}$, solid box

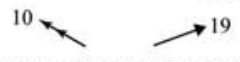

intersection lineations; L0x1, double barbed; L1 2 2, single barbed

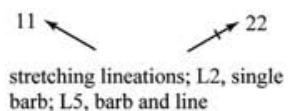
barb; L5, barb and line

FOLDS

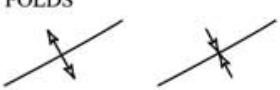

antiform (left) and synform (right)

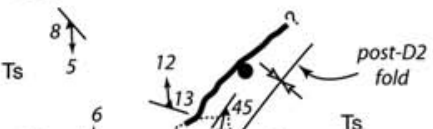

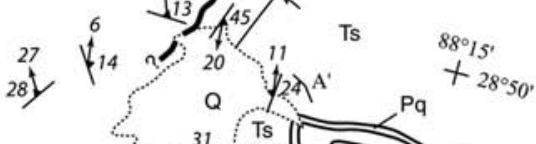
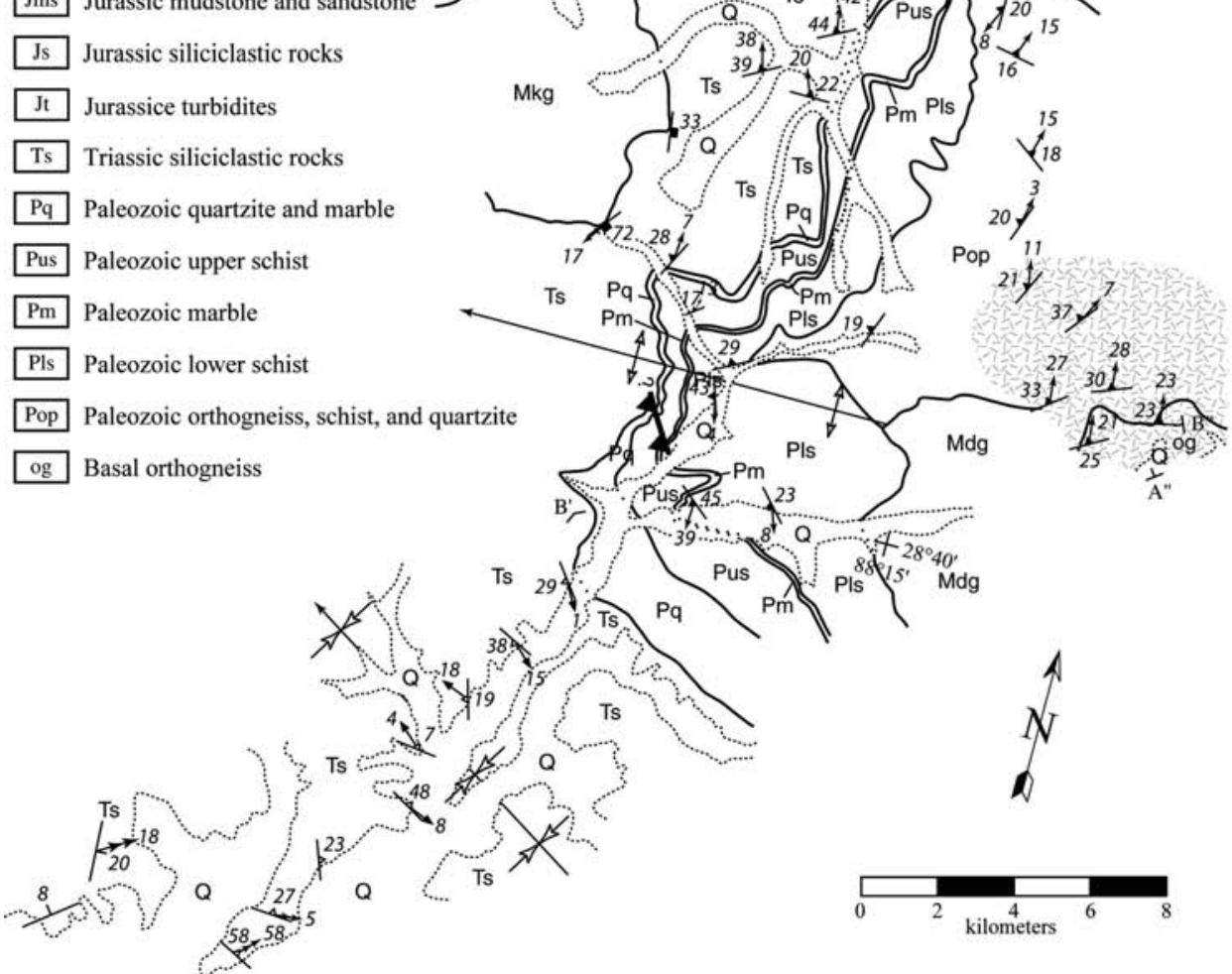

$\mathrm{B}^{\mathrm{X}}$

Fig. 2. Simplified geological map of Mabja Dome. Modified from Lee et al. (2004). 


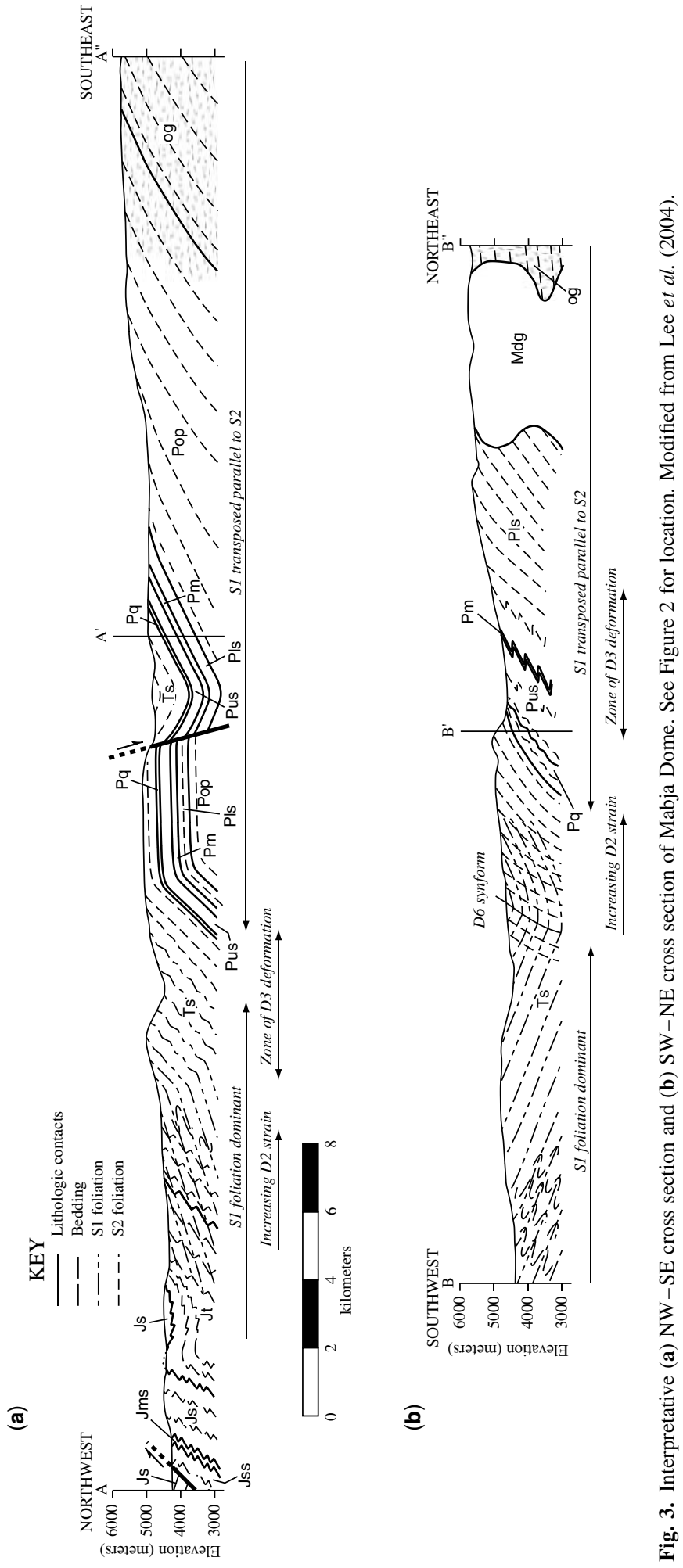


deformed pegmatite and aplite dyke swarm, two undeformed biotite + muscovite granites, and an undeformed rhyolite porphyry dyke. Local, penetratively deformed, decimetre-wide amphibolite dykes, containing hornblende + plagioclase \pm epidote \pm garnet, are interlayered with schist and orthogneiss of unit Pop. A coarse- to mediumgrained porphyritic to equigranular muscovite + $\mathrm{K}$-feldspar + quartz \pm biotite \pm garnet-bearing pegmatite and fine-grained leucocratic aplite dyke swarm constitutes as much as $30-35 \%$ of the lower half of units Pop and og; this swarm first appears within the kyanite zone, and dramatically increases in volume downward toward the sillimanite zone (Figs 2 \& 3). Two undeformed, medium- to coarse-grained, porphyritic two-mica granites, informally referred to as the Donggong and Kouwu granites (units Mdg and Mkg, respectively) were emplaced at deep structural levels into units Pls, Pop and og, and at shallower structural levels into unit Ts (Figs 2 \& 3). Finally, an undeformed rhyolite porphyry dyke was emplaced across Ts and, in part, along the contact between the Kouwu granite and unit Ts.

\section{Structural history}

The Mabja Dome preserves evidence for three major deformational events, two older penetrative subhorizontal contractional and subhorizontal stretching events, and a younger doming event (Lee et al. 2004). D1, the oldest deformational event, is best exposed and dominant at the highest structural levels, exhibits bedding horizontally shortened into map- to mesoscopic scale, upright to inclined, open to tight, typically disharmonic F1 folds. At higher structural levels, the axial planar foliation to these folds, S1, is a spaced, pressure solution or crenulation cleavage that with increasing structural depth becomes a penetrative fine-grained slaty cleavage and finally a somewhat coarser-grained phyllitic cleavage.

Superimposed on D1 structural fabrics is D2 a high-strain deformational event that is manifested at higher structural levels as S2, a closely spaced to weakly penetrative crenulation cleavage developed at high angles to S1. S2 changes with increasing depth from a spaced axial planar cleavage, to open to tight folds of $S 1$, to a penetrative axial planar cleavage, to isoclinal folds of S1. At structural levels below the garnet-in isograd, bedding and the $\mathrm{S} 1$ foliation have been transposed parallel to a mylonitic S2 foliation. Associated with the high-strain $\mathrm{S} 2$ foliation is a $c$. north-south stretching lineation, Ls2. The S2 mylonitic foliation is parallel to lithologic contacts and dips moderately NW on the NW flank of the dome and moderately
SW on the SW flank of the dome defining the domal geometry (Figs 2 \& 3) (Lee et al. 2004).

Meso- and microscopic structures, such as strain shadows on porphyroblasts, tails on K-feldspar porphyroclasts, oblique quartz grain-shape foliations, shear bands, and small (centimetre-scale) normal faults, record the sense of shear associated with, and after development of, the high-strain S2 foliation within orthogneiss and metasedimentary rocks (Lee et al. 2004). Lee et al. (2004) suggested that the bulk shear strain history changes from dominantly coaxial during the high temperature, main phase of D2 deformation to dominantly top$\mathrm{S}$ sense of shear during the low temperature, late phase of D2 deformation.

Subsequent to formation of D2 fabrics, the S2 foliation was domed into a doubly plunging, north-south elongate antiformal dome. The S2 mylonitic foliation dips moderately outward from the centre of the dome on the north, west and south flanks (Figs $2 \& 3$ ). Brittle structures are scarce and limited to two thrust faults of minor offset (tens of metres) and a 400-500 m dip-slip offset normal fault (Lee et al. 2004).

\section{Metamorphic history}

Microstructural textures indicate that peak metamorphism occurred after D1 deformation and prior to or during the D2 deformation. Peak metamorphism is defined by a prograde sequence of mineral assemblages that define a series of isograds (chloritoid-, garnet-, kyanite-, staurolite-, and sillimanite-in isograds) that increase towards the centre of the dome, are roughly concentric to the domal structure defined by the warped stratigraphy, and are parallel to the lithologic contacts and the S2 foliation. Based on mineral assemblages and quantitative thermobarometry, Lee et al. (2004) inferred temperatures and pressures of $c .475-530^{\circ} \mathrm{C}$ and c. $150-450 \mathrm{MPa}$ for the chloritoid-zone and calculated temperatures that increase from $575 \pm 50^{\circ} \mathrm{C}$ in the garnet zone to $705 \pm 65^{\circ} \mathrm{C}$ in the sillimanite zone and pressures from garnet-, stauroliteand sillimanite-zone rocks that are constant at c. $800 \mathrm{MPa}$, regardless of structural depth.

Four important observations are apparent in the metamorphic petrology results (Lee et al. 2004). (1) The presence of pressures as high as $800 \mathrm{MPa}$ (implying depths of $c .30 \mathrm{~km}$ ) suggests that these rocks were thickened or buried. (2) Based on PT determinations outlined above, apparent isotherms can be drawn in which temperatures increase with structural depth, yielding a metamorphic field gradient of $10-60^{\circ} \mathrm{C} / \mathrm{km}$. (3) The apparent gradient in pressure between the chloritoid-in isograd and garnet-zone rocks is greater than expected, 
indicating that these rocks were vertically thinned by c. $25-10 \%$ (horizontal stretching by a factor of $c$. four to ten). (4) Sillimanite-zone rocks exposed at the deepest structural levels yield calculated metamorphic pressures that are the same as, but higher temperatures than, garnet- and kyanitezone rocks at shallower structural levels. Lee et al. (2004) interpreted this to indicate that peak pressures and temperatures occurred asynchronously, such that garnet-zone rocks reached peak metamorphic conditions at c. $30 \mathrm{~km}$ depth before kyanite-zone rocks reached peak conditions at the same depth, which in turn reached peak conditions before sillimanite-zone rocks, again at the same depth.

In summary, the Mabja Dome records two penetrative deformations, the second characterized by horizontal stretching and vertical thinning. At the deepest structural levels, emplacement of a pegmatite and aplite dyke swarm, development of migmatites, and initiation of doming was synchronous with ductile stretching. Lee et al. (2004) attributed development of migmatites to thermal re-equilibration and adiabatic decompression during regional extensional collapse, possibly enhanced by an unexposed granitic pluton beneath the core of the dome.

\section{Geochronology and thermochronology}

\section{$U-P b$ geochronology}

To determine the timing of the high-strain D2 deformational event, $\mathrm{U}-\mathrm{Pb}$ geochronology by conventional thermal ionization mass spectrometry (TIMS) and sensitive high resolution ion microprobe (SHRIMP) techniques was completed on zircons and monazite from the post-tectonic Kouwu granite, and SHRIMP zircon analyses were performed on syn- to late-tectonic pegmatite dykes (Fig. 4, Table 1). Zircon and monazite were separated from $1-3 \mathrm{~kg}$ samples by standard gravity and magnetic techniques. Grains were hand-picked under alcohol for clarity, and lack of inclusions and cracks. TIMS analyses were performed on multigrain zircon and monazite fractions from the Kouwu granite. The fractions were spiked with a ${ }^{205} \mathrm{~Pb}$ tracer solution and analysed at the TIMS facility at University of California, Santa Barbara, following procedures outlined in McClelland \& Mattinson (1996). All of the TIMS zircon analyses are strongly discordant indicating the presence of significant inherited components in the multigrain fractions (Fig. 4). The SHRIMP technique was employed to improve spatial resolution and establish emplacement ages for the Kouwo granite and pegmatite dyke samples. Zircons selected for SHRIMP analysis were mounted in epoxy and polished to expose grain centres. Cathodoluminesence (CL) images were used to characterize the grains and select spots for analysis (Fig. 5). Zircons were analysed for $\mathrm{U}-\mathrm{Pb}$ on the SHRIMP-reverse geometry (RG) instrument at the Stanford University-United States Geological Survey Microanalytical Center (Palo Alto, California). A $30 \mu \mathrm{m}$ diameter spot size was used for all analyses. The analytical routine followed Williams (1998) and data reduction utilized the SQUID program of Ludwig (2001).

Zircons were analysed from sample MD71, a two-mica pegmatite that is part of the pegmatite and aplite dyke swarm, is concordant to the S2 mylonitic foliation, does not possess a mesoscopic penetrative fabric, but does possess ribbon grains in thin section. These relations suggest that this dyke was emplaced syn- to late during D2 deformation. Zircons from this sample consist of oscillatory zoned cores surrounded by high U rims and tips (Fig. 5a). Cores yield older ages representative of inherited components. Excluding three older tip analyses interpreted to record mixing of inherited and new zircon and the youngest analysis interpreted to record younger disturbance, the tips yield a weighted ${ }^{206} \mathrm{~Pb} /{ }^{238} \mathrm{U}$ mean age of $23.1 \pm 0.8 \mathrm{Ma}$ with a mean square of weighted deviate (MSWD) of 3.8 , or $23.1 \pm 1.6 \mathrm{Ma}$ incorporating the uncertainty introduced by a MSWD $=3.8$ (Fig. 4a). Penetrative D2 deformation was either continuing or was in its waning stages at these structural depths by this time.

Zircons from the undeformed Kouwu granite sample (MD86) contain inherited cores as well, as clearly indicated by the TIMS data (Figs $4 \mathrm{~b}$, c \& 5b). Excluding four older rim and tip analyses interpreted to record mixing of inherited and new zircon and two younger analyses interpreted to record younger disturbance, tips from this sample yield a weighted ${ }^{206} \mathrm{~Pb}-{ }^{238} \mathrm{U}$ mean age of $14.2 \pm 0.2 \mathrm{Ma}(\mathrm{MSWD}=0.5)$. A TIMS analysis of a single monazite yielded a $\mathrm{U}-\mathrm{Pb}$ age of $14.5 \pm 0.1 \mathrm{Ma}$. The TIMS monazite age is slightly older than the SHRIMP zircon age, perhaps indicating unresolved complexity in the monazite population. Our age for the Kouwu granite is not significantly different from the $14.4 \pm 0.1 \mathrm{Ma}$ age reported by Zhang et al. (2004). These data indicate that by $14.0-14.6 \mathrm{Ma}$, penetrative $\mathrm{D} 2$ deformation had ceased.

\section{${ }^{40} \mathrm{Ar} /{ }^{39} \mathrm{Ar}$ and apatite fission track thermochronology}

${ }^{40} \mathrm{Ar} /{ }^{39} \mathrm{Ar}$ and fission track were measured to characterize the exhumation history of the 
(a)
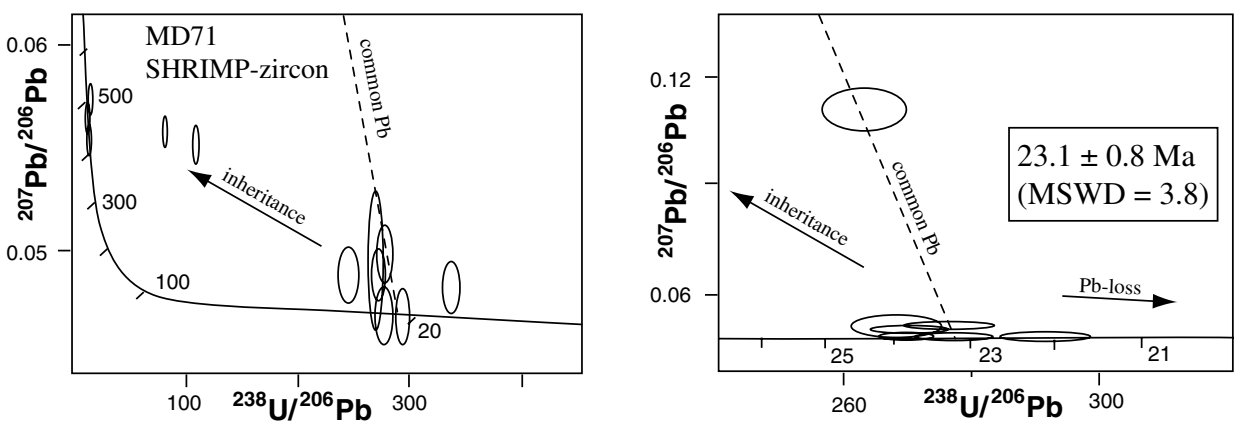

(b)
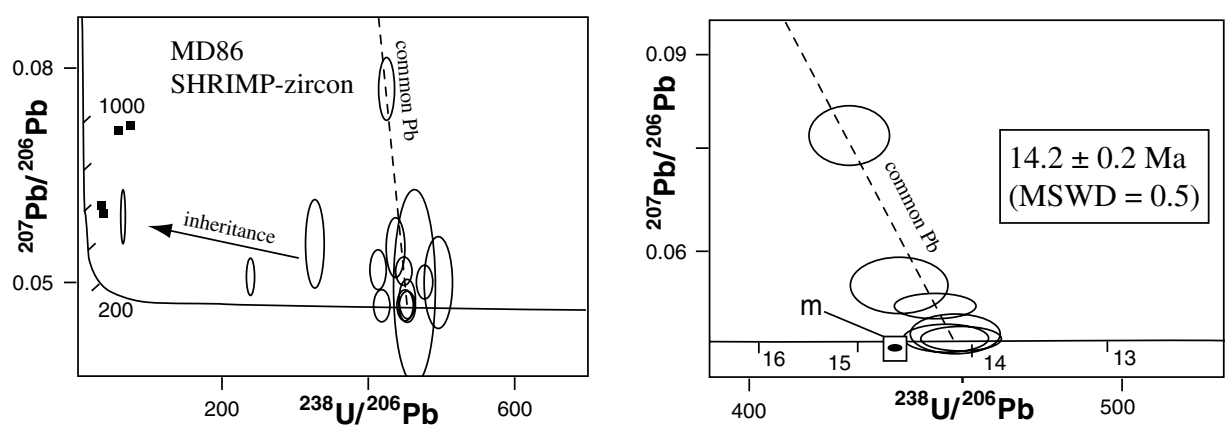

(c)

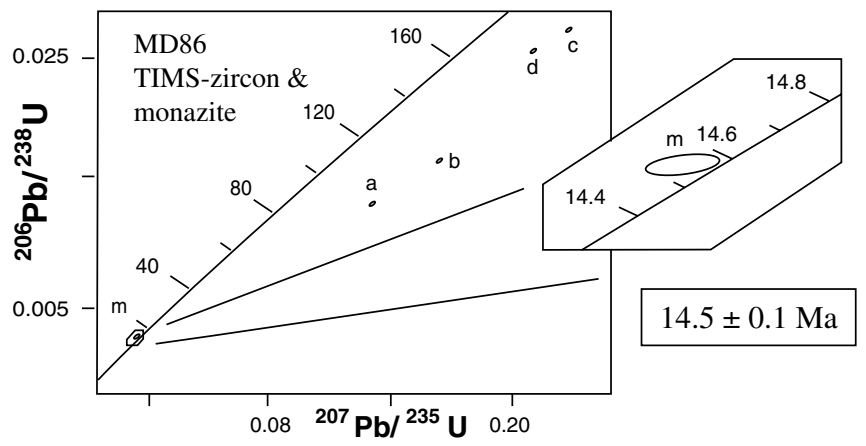

Fig. 4. (a) Left, U-Pb Tera-Wasserburg concordia plot of all zircon data from sample MD71, a pegmatite dyke (SHRIMP analyses 9, 13 and 16 are not plotted). Right, plot of rim data used to calculate weighted mean ${ }^{206} \mathrm{~Pb}-{ }^{238} \mathrm{U}$ age. (b) Left, U-Pb concordia plot of all zircon data from sample MD86, the undeformed Kouwu two-mica granite. Location of TIMS analyses marked with squares ( $2 \sigma$ ellipses are much smaller than squares). Right, plot of rim data used to calculate weighted mean ${ }^{206} \mathrm{~Pb}-{ }^{238} \mathrm{U}$ age (SHRIMP analysis 1 not plotted). TIMS monazite analysis plotted as solid ellipse ( $2 \sigma)$ within square and denoted by ' $\mathrm{m}$ '. (c) $\mathrm{U}-\mathrm{Pb}$ Wetherill concordia plot of TIMS zircon and monazite data from sample MD86. See Figure 8 for location of samples. U-Pb plots were generated using Ludwig (1999).

high-grade rocks within the Mabja Dome. Two hornblende, 25 mica and eight potassium feldspar samples collected from deformed amphibolites, orthogneisses, schists and pegmatites, and undeformed two-mica granites were analysed by ${ }^{40} \mathrm{Ar} /{ }^{39} \mathrm{Ar}$ methods. Seven apatite samples collected from orthogneisses, pegmatites and two-mica granites were analysed for fission track 
Table 1. $U-P b$ geochronologic data and apparent ages

\begin{tabular}{|c|c|c|c|c|c|c|c|c|}
\hline \multicolumn{9}{|c|}{ A. SHRIMP U-Pb data } \\
\hline Spot & $\begin{array}{c}\mathrm{U} \\
(\mathrm{ppm})\end{array}$ & $\begin{array}{c}\text { Th } \\
(\mathrm{ppm})\end{array}$ & $\mathrm{Th} / \mathrm{U}$ & $\begin{array}{l}{ }^{206} \mathrm{~Pb}^{*} \\
(\mathrm{ppm})\end{array}$ & $\mathrm{f}^{206} \mathrm{~Pb}_{\mathrm{c}}$ & ${ }^{238} \mathrm{U} /{ }^{206} \mathrm{~Pb}^{*}$ & ${ }^{207} \mathrm{~Pb} /{ }^{206} \mathrm{~Pb}^{*}$ & $\begin{array}{c}{ }^{206} \mathrm{~Pb} /{ }^{238} \mathrm{U}^{*} \\
\text { (Ma) }\end{array}$ \\
\hline \multicolumn{9}{|c|}{ Sample MD-71 } \\
\hline $1 \mathrm{c}$ & 620 & 97 & 0.16 & 42 & 0.02 & $12.56(1.5)$ & 0.0558 & $495(8)$ \\
\hline $2 \mathrm{c}$ & 1730 & 28 & 0.02 & 102 & 0.2 & $14.52(1.6)$ & $0.0573(0.7)$ & 428 (7) \\
\hline $3 \mathrm{c}$ & 2860 & 43 & 0.02 & 172 & 0.3 & $14.28(1.5)$ & $0.0580(0.7)$ & $435(6)$ \\
\hline $4 \mathrm{t}$ & 5490 & 167 & 0.03 & 17 & 0.3 & 270.51 & 0.0488 & ${ }^{\#} 23.7 \quad(0.4)$ \\
\hline $5 \mathrm{t}$ & 5990 & 144 & 0.02 & 19 & 0.4 & 267.84 (1.6) & $0.0495(4.5)$ & ${ }^{\#} 23.9(0.4)$ \\
\hline $6 \mathrm{t}$ & 5810 & 247 & 0.04 & 20 & 0.3 & $243.51(2.6)$ & $0.0488(1.8)$ & $26.3(0.7)$ \\
\hline $7 \mathrm{t}$ & 3840 & 166 & 0.04 & 30 & 1.0 & 109.14 & $0.0552(1.2)$ & $58.2(0.9)$ \\
\hline $8 \mathrm{t}$ & 5300 & 662 & 0.13 & 16 & 0.4 & $276.41(1.7)$ & $0.0499(1.9)$ & ${ }^{\#} 23.2(0.4)$ \\
\hline $9 \mathrm{t}$ & 4160 & 273 & 0.07 & 14 & 8.2 & 263.31 & 0.1115 & ${ }^{\#} 22.4(0.4)$ \\
\hline $10 \mathrm{t}$ & 6100 & 377 & 0.06 & 16 & 0.2 & $336.06(1.5)$ & 0.0482 & $19.1(0.3)$ \\
\hline $11 \mathrm{t}$ & 8450 & 1470 & 0.18 & 89 & 1.1 & $81.10(1.7)$ & $0.0560(0.7)$ & $78.2(1.3)$ \\
\hline $12 \mathrm{t}$ & 5090 & 527 & 0.11 & 15 & 0.05 & $291.59(1.7)$ & $0.0468(1.9)$ & ${ }^{\#} 22.1 \quad(0.4)$ \\
\hline $13 \mathrm{c}$ & 610 & 482 & 0.82 & 131 & 0.8 & $3.98(1.5)$ & $0.0977(0.5)$ & $1434(21)$ \\
\hline $14 \mathrm{c}$ & 250 & 184 & 0.77 & 16 & 0.2 & $13.14(1.6)$ & $0.0582(1.7)$ & 472 (7.4) \\
\hline $15 \mathrm{t}$ & 510 & 153 & 0.03 & 16 & 0.05 & $274.88(2.1)$ & $0.0469(2.0)$ & ${ }^{\#} 23.4(0.5)$ \\
\hline $16 \mathrm{t}$ & 4460 & 113 & 0.03 & 16 & 6.1 & $238.56(1.6)$ & $0.0947(5.3)$ & $25.3(0.4)$ \\
\hline \multicolumn{9}{|c|}{ Sample MD86 } \\
\hline $1 \mathrm{r}$ & 110 & 1 & 0.01 & 0.2 & 0.4 & $464.69(4.3)$ & $0.0495(18.2)$ & ${ }^{\#} 13.8(0.6)$ \\
\hline $2 \mathrm{c}$ & 1440 & 220 & 0.16 & 101 & 0.004 & $12.29(1.5)$ & $0.0574(0.9)$ & $504(8)$ \\
\hline $3 r$ & 350 & 1 & 0.00 & 0.9 & 1.1 & $327.36(2.4)$ & $0.0555(7.4)$ & $19.4(0.5)$ \\
\hline $4 \mathrm{r}$ & 1350 & 23 & 0.02 & 5 & 0.6 & 239.03 (1.7) & $0.0509(3.4)$ & $26.8(0.5)$ \\
\hline $5 \mathrm{t}$ & 5480 & 92 & 0.02 & 10 & 0.7 & 449.89 (1.6) & 0.0516 & ${ }^{\#} 14.2(0.2)$ \\
\hline $6 \mathrm{t}$ & 4190 & 257 & 0.06 & 8 & 0.5 & 478.99 (1.7) & $0.0500(3.0)$ & $13.4(0.2)$ \\
\hline $7 \mathrm{t}$ & 3780 & 64 & 0.02 & 8 & 3.9 & 426.67 (1.7) & $0.0772(3.8)$ & ${ }^{\#} 14.5 \quad(0.2)$ \\
\hline $8 \mathrm{t}$ & 470 & 19 & 0.04 & 0.8 & 0.5 & $498.85(2.5)$ & $0.0502(8.3)$ & $12.8(0.3)$ \\
\hline $9 \mathrm{t}$ & 2740 & 86 & 0.03 & 6 & 0.7 & 414.03 (1.7) & 0.0519 & $15.4(0.3)$ \\
\hline $10 \mathrm{r}$ & 370 & 29 & 0.08 & 5 & 1.4 & $64.79(2.4)$ & $0.0594(3.9)$ & $97(2.3)$ \\
\hline $11 \mathrm{t}$ & 1820 & 22 & 0.01 & 3 & 0.2 & 455.04 & 0.0476 & ${ }^{\#} 14.1 \quad(0.3)$ \\
\hline $12 \mathrm{t}$ & 5500 & 101 & 0.02 & 10 & 0.1 & $456.52(1.6)$ & $0.0469(2.5)$ & ${ }^{\#} 14.1 \quad(0.2)$ \\
\hline $13 \mathrm{t}$ & 1180 & 76 & 0.07 & 2 & 1.1 & 440.09 (1.9) & $0.0549(5.0)$ & ${ }^{\#} 14.5(0.3)$ \\
\hline $14 \mathrm{t}$ & 4550 & 86 & 0.02 & 9 & 0.1 & 452.85 (1.6) & $0.0469(2.7)$ & ${ }^{\#} 14.2(0.2)$ \\
\hline $15 \mathrm{r}$ & 3090 & 89 & 0.03 & 6 & 0.05 & 419.98 (1.7) & $0.0467(3.2)$ & $15.3(0.3)$ \\
\hline
\end{tabular}

Zircon SHRIMP analyses were performed on the SHRIMP-RG ion microprobe at the Stanford-United States Geological Survey Microanalytical Center at Stanford University. Spot abbreviations: number $=$ grain number; $\mathrm{c}=\mathrm{core} ; \mathrm{r}=\mathrm{rim} ; \mathrm{t}=$ tip.

$\mathrm{Pb}^{*}$, radiogenic $\mathrm{Pb} ; \mathrm{Pb}_{\mathrm{c}}$, common $\mathrm{Pb} ; \mathrm{f}^{206} \mathrm{~Pb}_{\mathrm{c}}=100\left({ }^{206} \mathrm{~Pb}_{\mathrm{c}} /{ }^{206} \mathrm{~Pb}_{\text {total }}\right)$.

${ }^{*}$ Calibration concentrations and isotopic compositions were based on replicate analyses of SL13 (238 ppm U) and R33 (419 Ma; Black et al. 2003). Reported SHRIMP ratios are not corrected for common Pb. Errors are reported in parentheses as percentages at the $1 \sigma$ level. SHRIMP ages were calculated from ${ }^{206} \mathrm{~Pb} /{ }^{238} \mathrm{U}$ ratios corrected for common $\mathrm{Pb}$ using the ${ }^{207} \mathrm{~Pb}$ method (see Williams 1998) and initial common Pb isotopic composition approximated from Stacey \& Kramers (1975). Uncertainties in millions of years reported as $1 \sigma$. Ages annotated with a hash sign (\#) were used in calculation of weighted mean ${ }^{206} \mathrm{~Pb} /{ }^{238} \mathrm{U}$ ages.

B. TIMS U-Pb data: sample MD-86

\begin{tabular}{|c|c|c|c|c|c|c|c|c|c|}
\hline $\begin{array}{l}\text { Fraction size } \\
(\mu \mathrm{m})\end{array}$ & $\begin{array}{l}\mathrm{Wt} \\
(\mathrm{mg})\end{array}$ & $\begin{array}{c}\mathrm{U} \\
(\mathrm{ppm})\end{array}$ & $\begin{array}{l}\mathrm{Pb}^{*} \\
(\mathrm{ppm})\end{array}$ & $\begin{array}{l}{ }^{206} \mathrm{~Pb} / \\
{ }^{204} \mathrm{~Pb}^{*}\end{array}$ & $\begin{array}{l}{ }^{206} \mathrm{~Pb} / \\
{ }^{207} \mathrm{~Pb}^{*}\end{array}$ & $\begin{array}{l}{ }^{206} \mathrm{~Pb} / \\
{ }^{208} \mathrm{~Pb}^{*}\end{array}$ & $\begin{array}{c}{ }^{206} \mathrm{~Pb} / \\
{ }^{238} \mathrm{U}^{\dagger} \\
(\mathrm{Ma})\end{array}$ & $\begin{array}{c}{ }^{207} \mathrm{~Pb} / \\
{ }^{235} \mathrm{U}^{\dagger}(\mathrm{Ma})\end{array}$ & $\begin{array}{c}{ }^{207} \mathrm{~Pb} / \\
{ }^{206} \mathrm{~Pb} \dagger(\mathrm{Ma})\end{array}$ \\
\hline a $50 \times 50 \times 80$ & 0.50 & 2037 & 27 & 25,485 & 13.7491 & 12.3650 & $85.3(0.2)$ & $126.4(0.3)$ & 991 (1) \\
\hline b $100 \times 100 \times 150$ & 0.26 & 1791 & 30 & 27,877 & 13.9128 & 10.9274 & $107.6(0.2)$ & $155.6(0.3)$ & 968 (1) \\
\hline
\end{tabular}


Table 1. Continued

B. TIMS U-Pb data: sample MD-86

\begin{tabular}{|c|c|c|c|c|c|c|c|c|c|}
\hline $\begin{array}{l}\text { Fraction size } \\
(\mu \mathrm{m})\end{array}$ & $\begin{array}{l}\mathrm{Wt} \\
(\mathrm{mg})\end{array}$ & $\begin{array}{c}\mathrm{U} \\
(\mathrm{ppm})\end{array}$ & $\begin{array}{c}\mathrm{Pb}^{*} \\
(\mathrm{ppm})\end{array}$ & $\begin{array}{l}{ }^{206} \mathrm{~Pb} / \\
{ }^{204} \mathrm{~Pb}^{*}\end{array}$ & $\begin{array}{l}{ }^{206} \mathrm{~Pb} / \\
{ }^{207} \mathrm{~Pb}^{*}\end{array}$ & $\begin{array}{l}{ }^{206} \mathrm{~Pb} / \\
{ }^{208} \mathrm{~Pb}^{*}\end{array}$ & $\begin{array}{c}{ }^{206} \mathrm{~Pb} / \\
{ }^{238} \mathrm{U}^{\dagger} \\
(\mathrm{Ma})\end{array}$ & $\begin{array}{c}{ }^{207} \mathrm{~Pb} / \\
{ }^{235} \mathrm{U}^{\dagger}(\mathrm{Ma})\end{array}$ & $\begin{array}{c}{ }^{207} \mathrm{~Pb} / \\
{ }^{206} \mathrm{~Pb} \dagger(\mathrm{Ma})\end{array}$ \\
\hline c $100 \times 100 \times 350$ & 0.63 & 1651 & 43 & 12,671 & 16.4590 & 8.5824 & $164.3(0.3)$ & $195.4(0.4)$ & $590(1)$ \\
\hline d $175 \times 175 \times 400$ & 0.60 & 1373 & 37 & 40,152 & 16.3649 & 14.6610 & $174.8(0.3)$ & $210.4(0.4)$ & $630(1)$ \\
\hline $\mathrm{m} 100 \times 300 \times 300$ & 0.20 & 3723 & 30 & 563 & 13.8179 & 0.3280 & $14.6(0.0)$ & $14.5(0.1)$ & $4(10)$ \\
\hline
\end{tabular}

TIMS analyses were performed at the University of California, Santa Barbara, following procedure described in McClelland \& Mattinson (1996). Zircon fractions were abraded to 30 to $60 \%$ of original mass and washed in warm $3 \mathrm{~N} \mathrm{HNO}_{3}$ and $3 \mathrm{~N} \mathrm{HCl}$ for 15 minutes each. All fractions were spiked with ${ }^{205} \mathrm{~Pb}-{ }^{235} \mathrm{U}$ tracer, and dissolved in a $50 \% \mathrm{HF} \gg 14 \mathrm{~N} \mathrm{HNO}_{3}$ solution (zircon) or $12 \mathrm{~N} \mathrm{HCl}$ (monazite) within $0.5 \mathrm{ml}$ Savillex ${ }^{\mathrm{TM}}$ capsules placed in $145 \mathrm{ml}$ TFE Teflon ${ }^{\mathrm{TM}}$ lined Parr acid digestion bomb. $\mathrm{Pb}$ and $\mathrm{U}$ were combined and loaded with $\mathrm{H}_{3} \mathrm{PO}_{4}$ and silica gel onto single degassed Re filaments. Isotopic compositions of $\mathrm{Pb}$ and $\mathrm{U}$ were determined through static collection on a Finnigan-MAT 261 multicollector mass spectrometer utilizing an ion counter for collection of the ${ }^{204} \mathrm{~Pb}$ beam.

Fraction: a, b, c, d designate conventional multigrain zircon fractions; $m$ designates monazite fraction. Zircon fractions are non-magnetic on Frantz magnetic separator at $1.8 \mathrm{~A}, 15^{\circ}$ forward slope, and side slope of $1^{\circ}$. Monazite fraction was magnetic on Frantz magnetic separator at $1.0 \mathrm{~A}, 20^{\circ}$ forward slope, and side slope of $10^{\circ}$.

$\mathrm{Pb}^{*}$, radiogenic $\mathrm{Pb}$

${ }^{*}$ Reported ratios corrected for fractionation $(0.125 \pm 0.038 \% / \mathrm{AMU})$ and spike $\mathrm{Pb}$. Ratios used in age calculation were adjusted for $2 \mathrm{pg}$ of blank $\mathrm{Pb}$ with isotopic composition of ${ }^{206} \mathrm{~Pb} /{ }^{204} \mathrm{~Pb}=18.6,{ }^{207} \mathrm{~Pb} /{ }^{204} \mathrm{~Pb}=15.5$, and ${ }^{208} \mathrm{~Pb} /{ }^{204} \mathrm{~Pb}=38.4,2 \mathrm{pg}$ of blank $\mathrm{U}$, $0.25 \pm 0.049 \% / \mathrm{AMU}$ fractionation for $\mathrm{UO}_{2}$, and initial common $\mathrm{Pb}$ with isotopic composition approximated from Stacey \& $\mathrm{Kramers}$ (1975) with an assigned uncertainty of 0.1 to initial ${ }^{207} \mathrm{~Pb} /{ }^{204} \mathrm{~Pb}$ ratio.

${ }^{t}$ Uncertainties reported as $2 \sigma$. Error assignment for individual analyses follows Mattinson (1987). An uncertainty of $0.2 \%$ is assigned to the ${ }^{206} \mathrm{~Pb} /{ }^{238} \mathrm{U}$ ratio based on estimated reproducibility unless this value is exceeded by analytical uncertainties. Calculated uncertainty in the ${ }^{207} \mathrm{~Pb} /{ }^{206} \mathrm{~Pb}$ ratio incorporates uncertainty due to measured ${ }^{204} \mathrm{~Pb} /{ }^{206} \mathrm{~Pb}$ and ${ }^{207} \mathrm{~Pb} /{ }^{206} \mathrm{~Pb}$ ratios, initial ${ }^{207} \mathrm{~Pb} /{ }^{204} \mathrm{~Pb}$ ratio, and composition and amount of blank. Decay constants used: ${ }^{238} \mathrm{U}=1.5513 \mathrm{E}-10,{ }^{235} \mathrm{U}=9.8485 \mathrm{E}-10,{ }^{238} \mathrm{U} /{ }^{235} \mathrm{U}=137.88$.

thermochronology. These samples were selected to provide age constraints on metamorphism and cooling histories across the dome along transects orientated approximately parallel and perpendicular to the Ls2 stretching lineation.
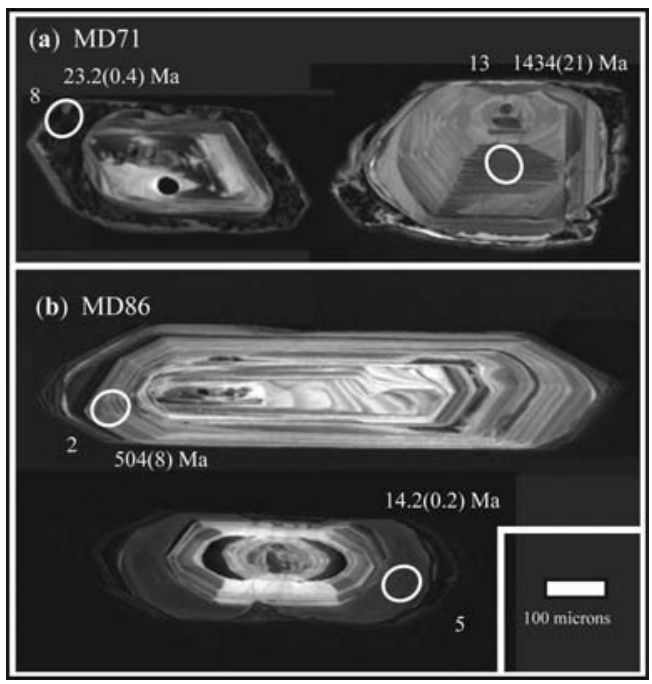

Fig. 5. Representative cathodoluminescence (CL) images of zircon analysed from samples (a) MD71 and (b) MD86. Ellipses indicate SHRIMP-RG analysis spots and the corresponding $\mathrm{U}-\mathrm{Pb}$ ages $( \pm 1 \sigma \mathrm{Ma})$.
For the ${ }^{40} \mathrm{Ar} /{ }^{39} \mathrm{Ar}$ samples discussed below, the estimated closure temperatures (assuming relatively rapid cooling rates) for hornblende, muscovite and biotite are $535 \pm 50^{\circ} \mathrm{C}$ (Harrison 1981), $370 \pm 50^{\circ} \mathrm{C}$ (Lister \& Baldwin 1996), and $335 \pm 50^{\circ} \mathrm{C}$ (Harrison et al. 1985; Grove \& Harrison 1996), respectively. For the low-temperature steps of potassium feldspar spectra, we use an estimated closure temperature of c. $200 \pm 50^{\circ} \mathrm{C}$ (Harrison et al. 1995; Lee 1995). Weighted mean plateau ages (WMPA) are reported where more than $50 \%$ of the ${ }^{39} \mathrm{Ar}$ released in three or more contiguous steps is within $2 \sigma$ error. For disturbed spectra, weighted mean ages (WMA) are reported where the spectrum is relatively flat, but does not meet the strict criteria for a plateau. The fission track age of a sample is usually interpreted as the time when the sample cooled below a closure temperature of $c \cdot 120-110^{\circ} \mathrm{C}$ (at a cooling rate of c. $10^{\circ} \mathrm{C} /$ million years) (e.g. Naeser 1979) and is determined by measuring the density of fission tracks and the U concentration of the sample (Naeser 1976).

Analytical techniques and $\mathrm{Ar} / \mathrm{Ar}$ data are available online at http://www.geolsoc.org.uk/ SUP18251. A hard copy can be obtained from the Society Library. Argon isotopic ages, and fission track analyses are provided in Tables 2 and 3; age spectra are shown in Figures 6 and 7, sample localities are shown in Figure 8, and ages are projected on approximately north-south and east-west cross-sections in Figure 9. 
Table 2. ${ }^{40} \mathrm{Ar} /{ }^{39} \mathrm{Ar}$ ages and calculated atmospheric ${ }^{40} \mathrm{Ar} /{ }^{36} \mathrm{Ar}$ ratios for muscovite and biotite samples

\begin{tabular}{|c|c|c|c|c|c|c|}
\hline Sample & $\begin{array}{l}\text { WMPA } \\
(\mathrm{Age} \pm \\
1 \sigma \mathrm{Ma})\end{array}$ & $\begin{array}{l}\text { WMA } \\
(\mathrm{Age} \pm \\
1 \sigma \mathrm{Ma})\end{array}$ & $\begin{array}{c}\text { TFA } \\
(\mathrm{Age} \pm \\
1 \sigma \mathrm{Ma})\end{array}$ & $\begin{array}{l}\text { Inverse Isochron } \\
\text { (Age } \pm \\
1 \sigma \mathrm{Ma})\end{array}$ & $\begin{array}{c}{ }^{40} \mathrm{Ar} /{ }^{36} \mathrm{Ar} \\
( \pm 1 \sigma)\end{array}$ & MSWD \\
\hline \multicolumn{7}{|c|}{ Muscovite } \\
\hline MD31B & - & $12.79 \pm 0.12$ & $12.85 \pm 0.12$ & $12.85 \pm 0.13$ & $293.6 \pm 4.3$ & $1.59<2.63$ \\
\hline MD48A & $14.03 \pm 0.14$ & - & $13.96 \pm 0.14$ & $14.01 \pm 0.14$ & $298.2 \pm 1.8$ & $1.63<2.63$ \\
\hline MD52 & $13.39 \pm 0.12$ & - & $11.56 \pm 0.13$ & $13.44 \pm 0.12$ & $289.3 \pm 3.6$ & $0.18<2.26$ \\
\hline MD79 & $16.77 \pm 0.15$ & - & $16.33 \pm 0.15$ & $16.79 \pm 0.16$ & $290.1 \pm 21.1$ & $1.23<2.63$ \\
\hline MD86 & $13.14 \pm 0.05$ & - & $13.09 \pm 0.05$ & $13.13 \pm 0.06$ & $296.0 \pm 5.7$ & $0.15<3.00$ \\
\hline MD93 & - & - & $14.96 \pm 0.14$ & $14.23 \pm 0.18$ & $333.0 \pm 9.0$ & $1.93<3.00$ \\
\hline MD97 & $13.54 \pm 0.05$ & - & $13.51 \pm 0.06$ & $13.54 \pm 0.06$ & $295.2 \pm 1.9$ & $0.71<2.41$ \\
\hline MD100 & - & $16.27 \pm 0.15$ & $16.44 \pm 0.15$ & $16.12 \pm 0.17$ & $325.2 \pm 19.6$ & $2.12<3.00$ \\
\hline MD47 & - & $16.99 \pm 0.15$ & $17.30 \pm 0.16$ & $17.09 \pm 0.19$ & $242.3 \pm 61.7$ & $0.02<3.83$ \\
\hline MD49A & $15.37 \pm 0.14$ & 二 & $15.52 \pm 0.14$ & $15.34 \pm 0.14$ & $307.1 \pm 11.2$ & $0.85<3.00$ \\
\hline MD55 & 二 & - & $14.47 \pm 0.13$ & $14.78 \pm 0.13$ & $274.8 \pm 3.1$ & $0.58<3.00$ \\
\hline MD71 & $13.48 \pm 0.12$ & - & $13.55 \pm 0.13$ & $13.48 \pm 0.13$ & $299.9 \pm 7.1$ & $0.97<2.63$ \\
\hline MD64A & $13.69 \pm 0.12$ & - & $13.66 \pm 0.12$ & $13.68 \pm 0.13$ & $295.6 \pm 1.6$ & $3.01<3.83$ \\
\hline MD69B & $3.33 \pm 0.06$ & - & $13.24 \pm 0.06$ & $13.29 \pm 0.09$ & $300.4 \pm 7.4$ & $1.52<3.00$ \\
\hline \multicolumn{7}{|l|}{ Biotite } \\
\hline MD33 & - & $15.51 \pm 0.14$ & $15.97 \pm 0.14$ & $15.52 \pm 0.14$ & $294.2 \pm 1.5$ & $1.08<2.15$ \\
\hline MD39 & - & $17.60 \pm 0.15$ & $17.71 \pm 0.16$ & $17.66 \pm 0.16$ & $287.0 \pm 3.0$ & $0.75<2.63$ \\
\hline MD48A & $15.37 \pm 0.14$ & - & $15.30 \pm 0.14$ & $15.34 \pm 0.22$ & $312.2 \pm 131.6$ & $1.48<3.00$ \\
\hline MD52 & $13.60 \pm 0.12$ & - & $13.57 \pm 0.12$ & $13.61 \pm 0.12$ & $292.7 \pm 4.3$ & $0.54<2.07$ \\
\hline MD79 & $15.88 \pm 0.15$ & - & $14.92 \pm 0.14$ & $16.89 \pm 0.75$ & $231.2 \pm 47.4$ & $0.16<3.83$ \\
\hline MD86 & - & $12.93 \pm 0.06$ & $13.00 \pm 0.06$ & $12.84 \pm 0.08$ & $340.0 \pm 23.5$ & $3.18<3.83$ \\
\hline MD97 & - & 二 & $13.71 \pm 0.06$ & $13.48 \pm 0.12$ & $288.5 \pm 29.3$ & $83.44>3.83$ \\
\hline MD37 & $13.49 \pm 0.12$ & - & $13.49 \pm 0.15$ & $13.58 \pm 0.13$ & $253.4 \pm 27.9$ & $0.35<3.00$ \\
\hline MD71 & - & $13.60 \pm 0.11$ & $13.39 \pm 0.11$ & $13.59 \pm 0.12$ & $297.3 \pm 11.4$ & $3.34<3.83$ \\
\hline MD64A & - & $13.79 \pm 0.13$ & $13.72 \pm 0.12$ & $13.86 \pm 0.13$ & $247.9 \pm 17.9$ & $2.20<3.00$ \\
\hline MD69A & - & $13.48 \pm 0.11$ & $13.46 \pm 0.11$ & $13.49 \pm 0.12$ & $292.2 \pm 14.9$ & $1.87<3.83$ \\
\hline
\end{tabular}

WMPA, weighted mean plateau age; WMA, weighted mean age; TFA, total fusion age; MSWD, mean square of weighted deviates.

Hornblende Hornblende samples MD41 and MD49B from penetratively deformed amphibolites within the kyanite zone were analysed to provide an estimate for the age of peak metamorphism.
Both samples yield disturbed age spectra with double-gradient-type patterns suggesting incorporation of excess Ar, and are uninterruptible (Fig. 6).

Table 3. Apatite fission track analyses

\begin{tabular}{lcccccccc}
\hline Sample & $\begin{array}{c}\text { No. } \\
\text { grains }\end{array}$ & $\begin{array}{c}\text { Standard } \\
\text { track density } \\
\left(\times 10^{6} \mathrm{~cm}^{-2}\right)\end{array}$ & $\begin{array}{c}\text { Fossil track } \\
\text { density } \\
\left(\times 10^{4} \mathrm{~cm}^{-2}\right)\end{array}$ & $\begin{array}{c}\text { Induced } \\
\text { track density } \\
\left(\times 10^{4} \mathrm{~cm}^{-2}\right)\end{array}$ & $\begin{array}{c}\text { Chi squared } \\
\text { prob. } \\
(\%)\end{array}$ & $\begin{array}{c}\text { Central } \\
\text { age } \\
(\mathrm{Ma})\end{array}$ & $\begin{array}{c}\text { Mean } \\
\text { track length } \\
(\mu \mathrm{m})\end{array}$ & $\begin{array}{c}\text { Std. } \\
\text { dev. } \\
(\mu \mathrm{m})\end{array}$ \\
\hline MD33 & 20 & $2.10(3354)$ & $2.03(13)$ & $82.5(825)$ & 94 & $8.3 \pm 2.3$ & & \\
MD39 & 20 & $2.10(3354)$ & $2.97(19)$ & $93.0(595)$ & 41 & $10.7 \pm 2.6$ & \\
MD52 & 25 & $2.08(3327)$ & $9.38(75)$ & $326.9(2615)$ & 76 & $9.5 \pm 1.2$ & \\
MD64 & 25 & $2.08(3327)$ & $4.75(38)$ & $187.8(1502)$ & 88 & $8.4 \pm 1.4$ & \\
MD71 & 30 & $2.07(3301)$ & $6.10(58)$ & $175.8(1672)$ & 61 & $11.5 \pm 1.6$ & \\
MD86 & 20 & $2.07(3301)$ & $16.6(69)$ & $598.3(2489)$ & 63 & $9.2 \pm 1.2$ & & \\
MD97 & 8 & $2.05(3274)$ & $14.9(31)$ & $50.3(1046)$ & 7 & $9.9 \pm 2.3$ & $14.4 \pm 0.1(65)$ & 0.9
\end{tabular}

All apatite separates were prepared and analysed by A. Blythe at the University of Southern California. All ages are central ages (Galbraith \& Laslett 1993). The conventional method (Green 1981) was used to determine errors on ages. Ages were calculated using zeta $=320 \pm 9$ for dosimeter glass SRM 962a (e.g. Hurford \& Green 1983). Numbers in parentheses represent total tracks counted or total lengths measured. The chi-squared test estimated the probability that individual grain ages for each sample belong to a single population with Poissonian distribution (Galbraith 1981). 

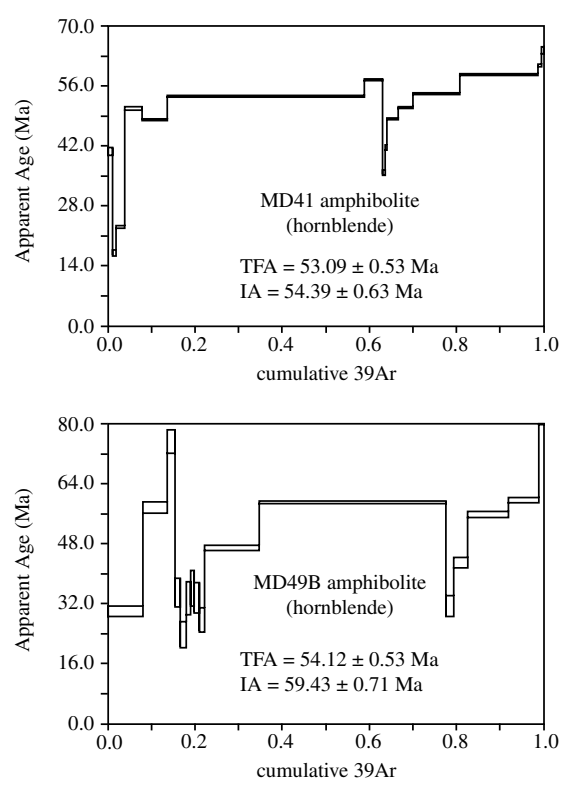

Fig. 6. Hornblende ${ }^{40} \mathrm{Ar} /{ }^{39} \mathrm{Ar}$ age spectra. Total fusion (TFA) and isochron (IA) ages given.

White mica Fourteen white mica samples from orthogneisses, schists, pegmatites and granites were analysed. Because most of the samples yield WMPA or WMA ages that are indistinguishable from their inverse isochron and/or total fusion ages and yield a trapped ${ }^{40} \mathrm{Ar} /{ }^{36} \mathrm{Ar}$ ratio that is not significantly different from the atmospheric ratio of 295.5 (Fig. 7, Table 2), we report their inverse isochron age (Figs 8 \& 9). White mica ages from schists and orthogneisses increase down structural section from $12.85 \pm 0.13 \mathrm{Ma}$ at the top of garnet-zone rocks to $17.09 \pm 0.19 \mathrm{Ma}$ at the bottom of garnet-zone rocks, and then decrease at the deepest structural levels to $13.29 \pm 0.09 \mathrm{Ma}$. This age pattern also holds true whether we report WMPA/WMA or total fusion ages. White micas from syn- to late-D2 pegmatites and post-tectonic two-mica granites yield ages of $c$. $13.4 \mathrm{Ma}$ (range of $13.13 \pm 0.06 \mathrm{Ma}$ to $13.54 \pm 0.06 \mathrm{Ma}$ ).

Biotite Like the muscovite samples, most biotites yield WMPA or WMA ages that are indistinguishable from their inverse isochron and total fusion ages, and yield a trapped ${ }^{40} \mathrm{Ar} /{ }^{36} \mathrm{Ar}$ ratio that is not significantly different from the atmospheric ratio of 295.5 (Fig. 7, Table 2); therefore, we report sample inverse isochron ages. Biotite ages also increase down structural section from $13.58 \pm 0.13 \mathrm{Ma}$ to $17.66 \pm 0.16 \mathrm{Ma}$, and then decrease at the deepest structural levels to $13.49 \pm 0.12$ Ma. Biotites from the post-tectonic two-mica granites yield ages of $12.84 \pm 0.08 \mathrm{Ma}$ to $13.61 \pm 0.12 \mathrm{Ma}$.

Potassium feldspar Seven potassium feldspar samples from orthogneiss, pegmatite and twomica granite were analysed (Fig. 7). Intermediatedepth orthogneiss samples MD33 and MD39 yield complex age spectra characterized by ages that climb steeply and erratically defining doublegradient patterns indicative of incorporation of excess argon. Ages older than $100 \mathrm{Ma}$ occur at the high-temperature steps; these spectra are uninterruptible and are not shown in Figure 7. Migmatite sample MD64A yields an age spectrum with old apparent ages over the first c. 5\% of ${ }^{39}$ Ar released, suggesting incorporation of excess argon. Over the next c. $20 \%$ of the 39Ar released, ages range from as young as $c .12 .7 \mathrm{Ma}$ to as old as $c .15 \mathrm{Ma}$, and then climb gradually to a maximum age of $c .17 .7$ Ma. Sample MD71, collected from a syn- to lateD2 pegmatite, yields a pattern indicative of excess argon in the first few low-temperature steps and then ages that climb slowly, but erratically, from c. $12.5 \mathrm{Ma}$ to $c$. $14.4 \mathrm{Ma}$ and then rapidly to 16.2 Ma over the last few high-temperature steps. Post-tectonic two-mica granite samples MD86 and MD97 yield simple spectra. At the lowest temperatures, these spectra exhibit little or no excess argon and low-temperature ages of $c .11 .1-11.3 \mathrm{Ma}$ that slowly climb to $c .13 .0-13.1 \mathrm{Ma}$ at high temperatures. Sample MD52 from a post-tectonic twomica granite yields a double-gradient age spectrum pattern similar to orthogneiss samples, suggesting incorporation of excess argon.

Apatite Seven apatite separates from orthogneiss, granite and a pegmatite were analysed to constrain the low-temperature exhumation history (Figs $7 \&$ 8). The fission track central ages (Galbraith \& Laslett 1993) for the seven samples range from $8.3 \pm 2.3$ to $11.5 \pm 1.6 \mathrm{Ma}$ with $1 \sigma$ uncertainty. The uncertainties are large on these ages because the apatites had relatively low concentrations of $\mathrm{U}$. The seven fission track ages overlap at $1 \sigma$ error, indicating that the dome uniformly cooled through $c .115^{\circ} \mathrm{C}$ at $9.5 \pm 0.6 \mathrm{Ma}$, the mean age for all samples. Sample MD97 yields a mean track length of $14.4 \pm 0.1(n=65)$ with a standard deviation of 0.9 indicating very rapid cooling.

\section{Significance of geochronologic and thermochronologic results}

Our $\mathrm{U}-\mathrm{Pb}$ zircon and monazite measurements on the syn- to late-D2 leucocratic dyke swarm and post-D2 two-mica granites yield emplacement ages of $23.1 \pm 0.8 \mathrm{Ma}$ and $c$. $14.0-14.6 \mathrm{Ma}$, respectively. These data indicate that high-strain 

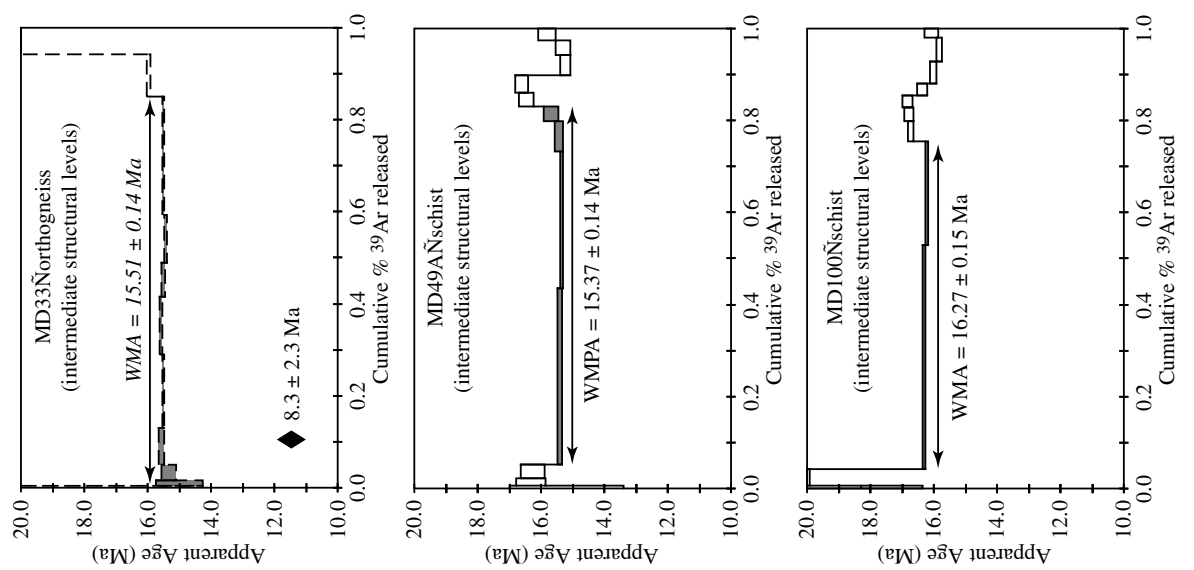

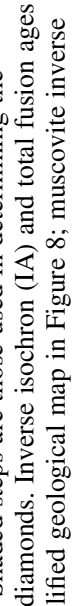
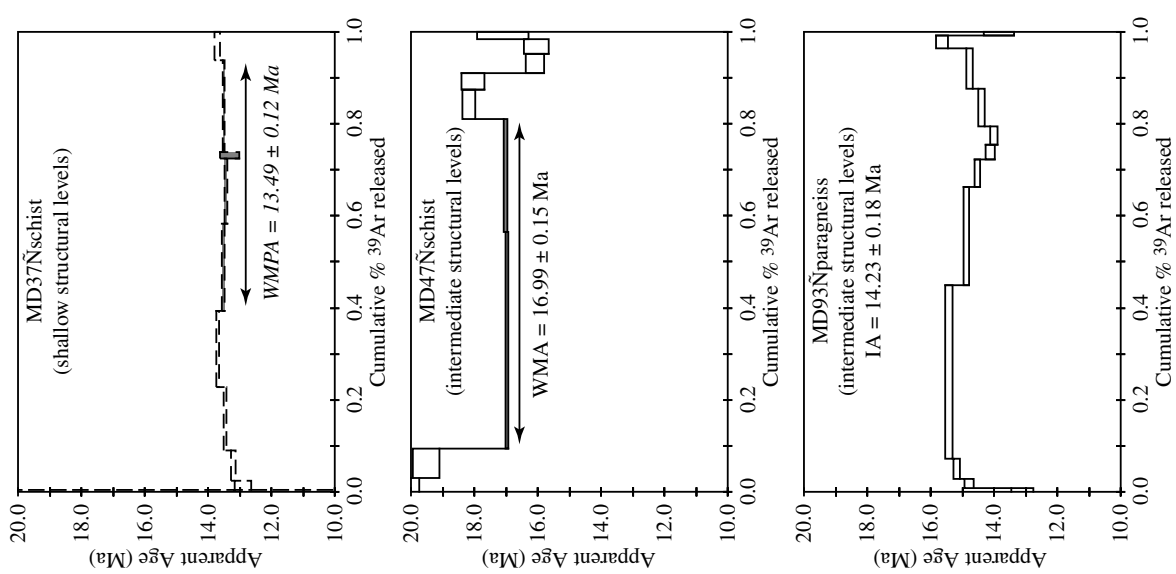

퓽 긍 है

至

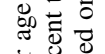

这莺 $\frac{0}{2}$

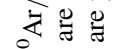

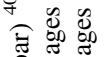

至兽总

恶

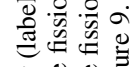

苋.气.气.

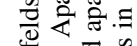

实灾

帘

용.
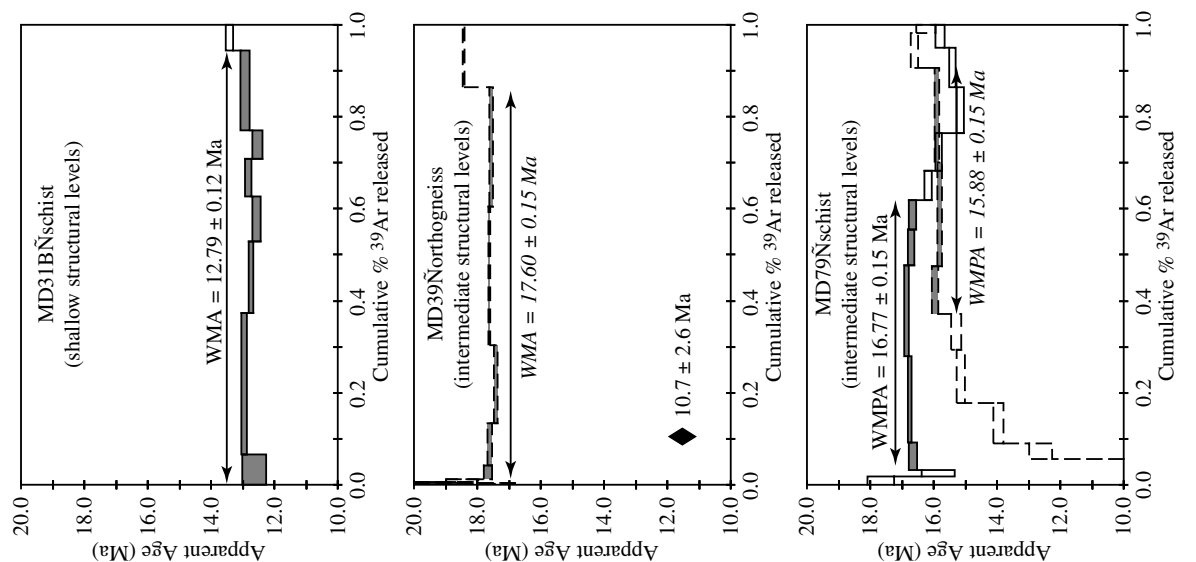

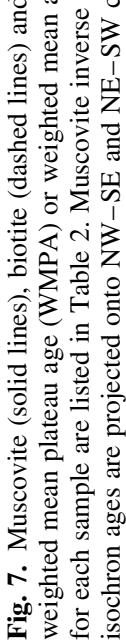



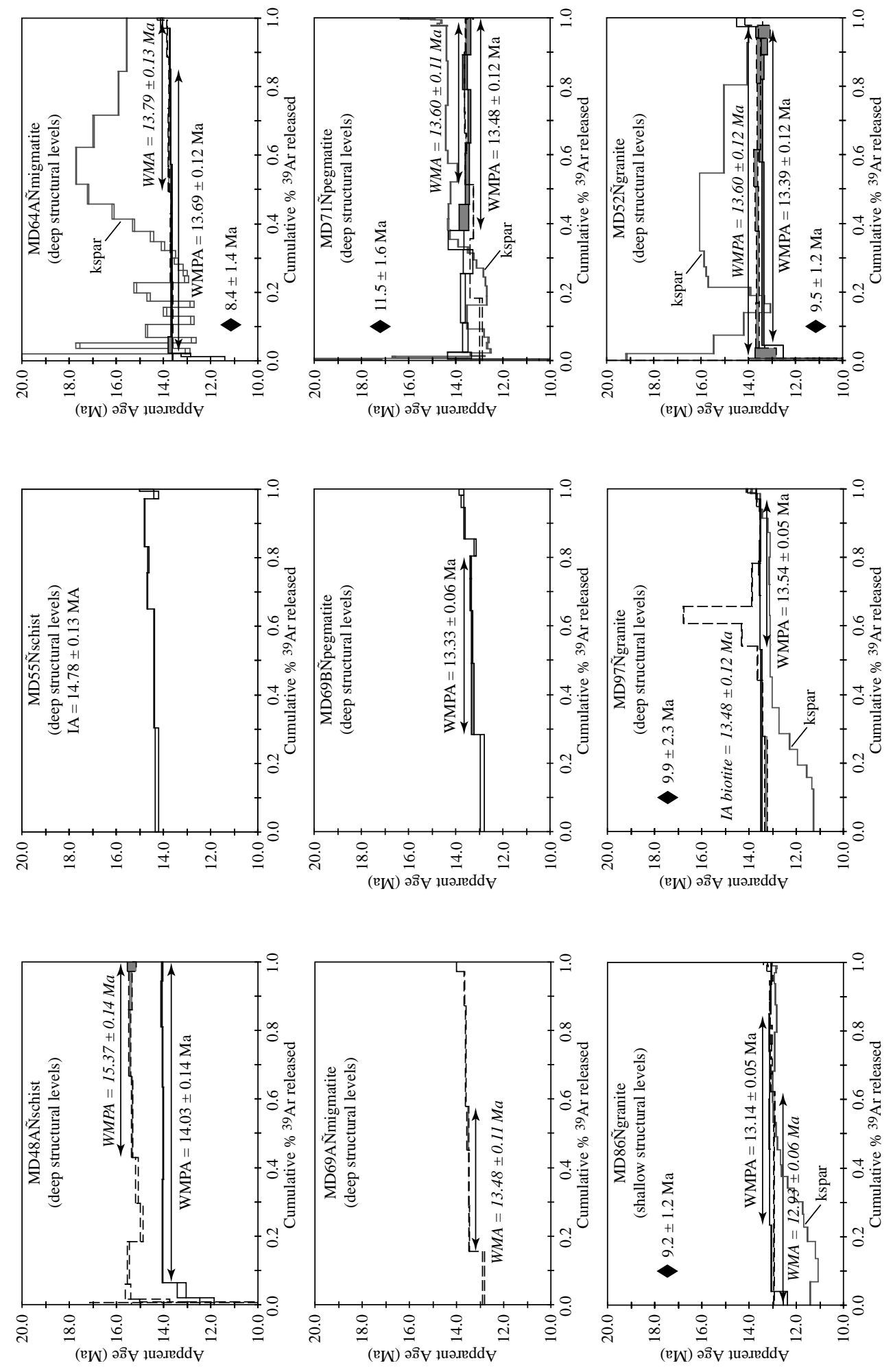

نัّ 


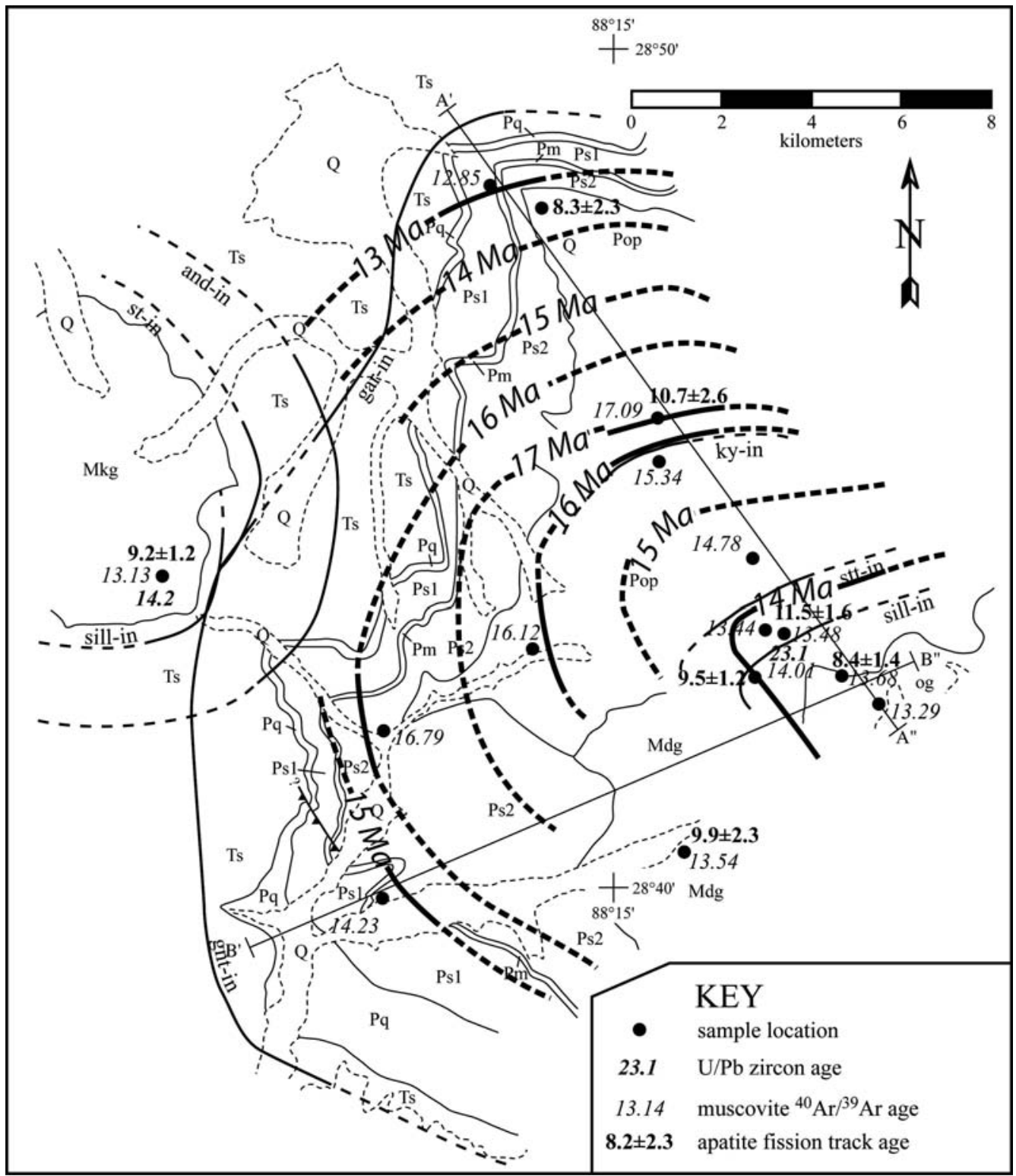

Fig. 8. Simplified geological map of the Mabja Dome showing location of geochronology and thermochronology samples, U-Pb zircon, muscovite ${ }^{40} \mathrm{Ar} /{ }^{39} \mathrm{Ar}$, and apatite fission track ages. Chrontours for muscovite ${ }^{40} \mathrm{Ar} /{ }^{39} \mathrm{Ar}$ inverse isochron ages from metamorphic and orthogneissic rocks shown as heavy lines; metamorphic isograds shown as thin lines. Solid and dashed lines indicate well-constrained and inferred locations, respectively, of chrontours and isograds. See Table 2 for muscovite inverse isochron ages and Figure 7 for age spectra.

D2 vertical thinning and horizontal stretching, synchronous with peak metamorphism and generation of migmatites, was continuing at 23.1 Ma and had ceased by $c .14 .3 \mathrm{Ma}$.

Our white mica ${ }^{40} \mathrm{Ar} /{ }^{39} \mathrm{Ar}$ ages from metamorphic rocks range from $17.09 \pm 0.19 \mathrm{Ma}$ to
$12.85 \pm 0.13 \mathrm{Ma}$, and define approximately concentric chrontours centred on the core of the dome (Figs $8 \& 9$ ). The estimated $370 \pm 50^{\circ} \mathrm{C}$ closure temperature for muscovite (Lister \& Baldwin 1996) is somewhat higher than the minimum temperature at which quartz will deform ductilely. 

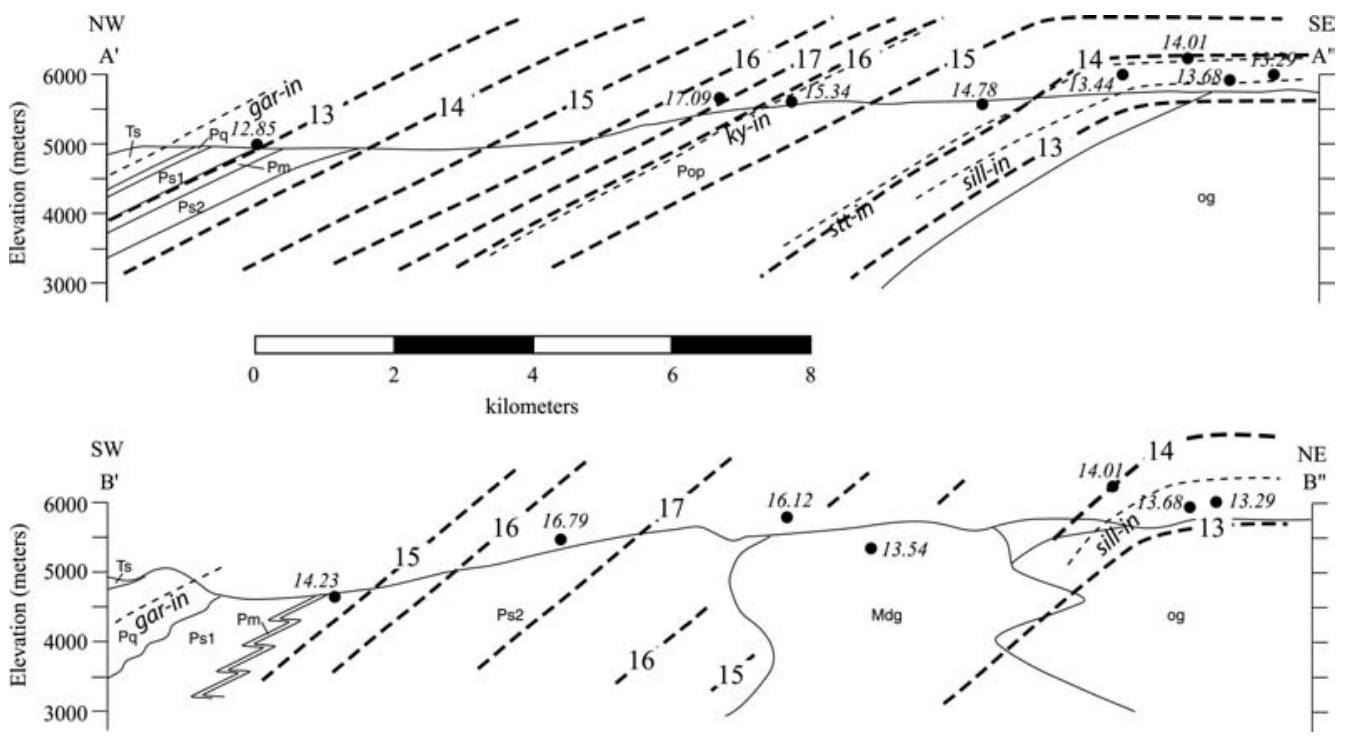

Fig. 9. Muscovite ${ }^{40} \mathrm{Ar} /{ }^{39} \mathrm{Ar}$ inverse isochron ages and chrontours (heavy lines), and metamorphic isograds (thin lines) projected onto the eastern parts of cross-sections $\mathrm{A}^{\prime}-\mathrm{A}^{\prime \prime}$ and $\mathrm{B}^{\prime}-\mathrm{B}^{\prime \prime}$ (see Figure 8). See Table 2 for muscovite inverse isochron ages and Figure 7 for age spectra.

Hence, D2 ductile deformation within Mabja Dome must have ended between 17 and $13 \mathrm{Ma}$, consistent with the $c$. 14.0-14.6 Ma emplacement age for the post-tectonic two-mica granite. White mica ${ }^{40} \mathrm{Ar} /{ }^{39} \mathrm{Ar}$ ages increase down structural section from $12.85 \pm 0.13 \mathrm{Ma}$ at the top of the garnet zone to $17.09 \pm 0.19 \mathrm{Ma}$ at the bottom of the garnet zone, and then decrease farther down structural section to $c$. $13.4 \mathrm{Ma}$ in staurolite-zone rocks and deeper (Figs 8 \& 9). At Kangmar Dome, Lee et al. (2000) documented an increase in mica ${ }^{40} \mathrm{Ar} /{ }^{39} \mathrm{Ar}$ age with structural depth, and attributed this to refrigeration from below due to underthrusting of a cold slab along the Gyirong-Kangmar thrust fault (GKT) (Fig. 1). Mabja Dome, like Kangmar Dome, lies in the hanging wall of the north-dipping GKT and, as for Kangmar, we suggest that the pattern of increasing mica ${ }^{40} \mathrm{Ar} /{ }^{39} \mathrm{Ar}$ ages with depth was caused by refrigeration of hot Mabja rocks by underthrusted cold Tethyan sediments. In contrast to Kangmar, ${ }^{40} \mathrm{Ar} /{ }^{39} \mathrm{Ar}$ white mica ages in Mabja decrease at the deepest structural levels. This observation, together with $\mathrm{U} / \mathrm{Pb}$ ages and field observations documenting that emplacement of the 14.0-14.6 Ma two-mica granites post-date D2 deformation, indicates that middle Miocene refrigeration at the deepest structural levels was likely overprinted by a reheating event at c. 14.3 Ma followed by rapid conductive cooling. Finally, low temperature potassium feldspar ${ }^{40} \mathrm{Ar} /{ }^{39} \mathrm{Ar}$ and apatite fission track data yield uniform ages demonstrating that the dome symmetrically cooled between $200 \pm 50^{\circ} \mathrm{C}$ and $115 \pm 5^{\circ} \mathrm{C}$ from $c$. $12.5 \mathrm{Ma}$ to $9.5 \pm 0.6 \mathrm{Ma}$ (Figs $7 \& 8$ ).

Calculated cooling rates across the dome based on our zircon and monazite $\mathrm{U}-\mathrm{Pb}$, mica and potassium feldspar ${ }^{40} \mathrm{Ar} /{ }^{39} \mathrm{Ar}$, and apatite fission track data are shown in Figure 10. The syn- to latetectonic pegmatite exhibits rapid average cooling rates of $40-60^{\circ} \mathrm{C} /$ million years following emplacement at $23.1 \pm 0.8 \mathrm{Ma}$ and a zircon closure temperature of $c .750^{\circ} \mathrm{C}$ (Cherniak \& Watson 2003), to a temperature of $115 \pm 5^{\circ} \mathrm{C}$ at $9.5 \pm 0.6 \mathrm{Ma}$ (Fig. 10). Initial rapid cooling of the pegmatite at a rate of $c .350^{\circ} \mathrm{C} /$ million years followed by slower cooling at $6^{\circ} \mathrm{C} /$ million years might be a more reasonable initial cooling history (Fig. 10). Shallow (garnet zone), intermediate (garnet and kyanite zone) and deep (sillimanite zone) structural levels also yield rapid cooling rates of $45-60^{\circ} \mathrm{C} /$ million years from $c$. $370-335^{\circ} \mathrm{C}$ to $120-110^{\circ} \mathrm{C}$ between $17.09 \pm 0.19 \mathrm{Ma}$ and $9.5 \pm 0.6 \mathrm{Ma}$ (Fig. 10). Finally, the two-mica granites cooled rapidly at rates of $c .350^{\circ} \mathrm{C} /$ million years from a zircon closure temperature of $c .750^{\circ} \mathrm{C}$ at $c$. 14.3 Ma to $c .370^{\circ} \mathrm{C}$ at $c .13 .4 \mathrm{Ma}$ (Fig. 10), suggesting conductive cooling and possibly exhumation. Continued cooling of the two-mica granites from $370 \pm 50^{\circ} \mathrm{C}$ at $c$. $13.4 \mathrm{Ma}$ to $115 \pm 5^{\circ} \mathrm{C}$ at $9.5 \pm 0.6 \mathrm{Ma}$ was slower, but still high, at a rate of $60^{\circ} \mathrm{C} /$ million years; this is essentially the same cooling rate and age range as documented in the metasedimentary and orthogneissic rocks. 


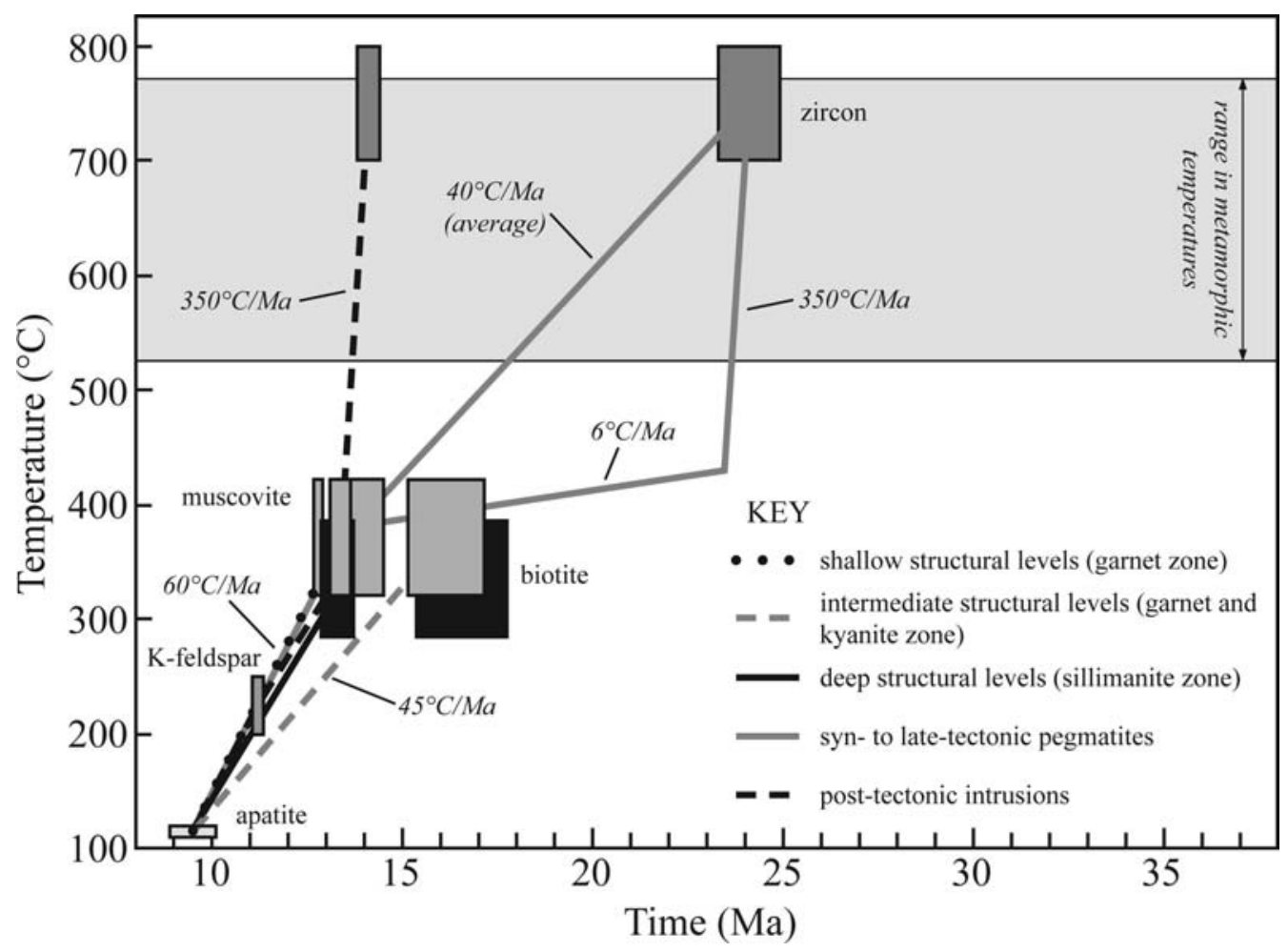

Fig. 10. Temperature-time plot showing cooling histories for geochronology and thermochronology samples from Mabja Dome.

Deep, intermediate and shallow structural levels, the pegmatite dyke swarm, and the posttectonic granite intrusions yield nearly identical rapid cooling rates of $c .45-60^{\circ} \mathrm{C} /$ million years from $370 \pm 50^{\circ} \mathrm{C}$ to $115 \pm 5^{\circ} \mathrm{C}$ between $17.09 \pm 0.19$ to $13.29 \pm 0.09 \mathrm{Ma}$ and $9.5 \pm 0.6$ $\mathrm{Ma}$, which are most likely the result of a combination of refrigeration and exhumation during thrust faulting and erosion. If we assume a linear, steady-state geothermal gradient of $30^{\circ} \mathrm{C} / \mathrm{km}$ for the crust, these cooling rates imply an exhumation rate of $c .1 .5-2.0 \mathrm{~mm} / \mathrm{a}$. This assumption is probably reasonable in areas of slow denudation, but may not be correct where the thermal structure of the crust has been perturbed by advection (Mancktelow \& Grasemann 1997; Moore \& England 2001), implying that the exhumation rate we calculate may be a minimum.

\section{Discussion}

\section{Formation of Mabja Gneiss Dome}

The mechanism by which Himalayan gneiss domes form is typically attributed to one or more of three processes: metamorphic core complex-type extension, diapirism, or duplex formation (Burg et al. 1984; Le Fort, 1986; Le Fort et al. 1987; Chen et al. 1990; Lee et al. 2000, 2004). Based on field, structural and metamorphic petrology data from Mabja, Lee et al. (2004) advocated a doming mechanism driven, at least in part, by buoyant migmatite diapirs generated during adiabatic decompression synchronous with D2 ductile extensional collapse and possibly enhanced by a proposed buoyant granitic body at depth. In contrast, Lee et al. (2000), building on the work of Burg et al. (1984) and Chen et al. (1990), concluded that the domal geometry in Kangmar was the result of hot middle crust thrust up and over a north-dipping ramp along the GKT; thermochronologic data were the key dataset to this interpretation. Without similar thermochronology, Lee et al. (2004) could not exclude the possibility that a similar regional contractional and/or extensional event contributed to the growth of the domal form in Mabja.

Our new ages, in conjunction with field, structural and metamorphic data from Lee et al. (2004) and regional field relations, provide new insight into the formation of Mabja Dome (Fig. 11). 
The S2 foliation, M1 isograds, and muscovite ${ }^{40} \mathrm{Ar} /{ }^{39} \mathrm{Ar}$ chrontours are domed (Figs 8 \& 9), whereas the low-temperature-step potassium feldspar ${ }^{40} \mathrm{Ar} /{ }^{39} \mathrm{Ar}$ and apatite fission track chrontours are not. This observation means that an episode of doming occurred at temperatures below $c .370^{\circ} \mathrm{C}$ (the estimated blocking temperature for muscovite) after c. $15 \mathrm{Ma}$, the youngest muscovite ${ }^{40} \mathrm{Ar} /{ }^{39} \mathrm{Ar}$ chrontour that appears to be domed, but above c. $200^{\circ} \mathrm{C}$ (the estimated blocking temperature for

(a)

\section{Early Miocene}

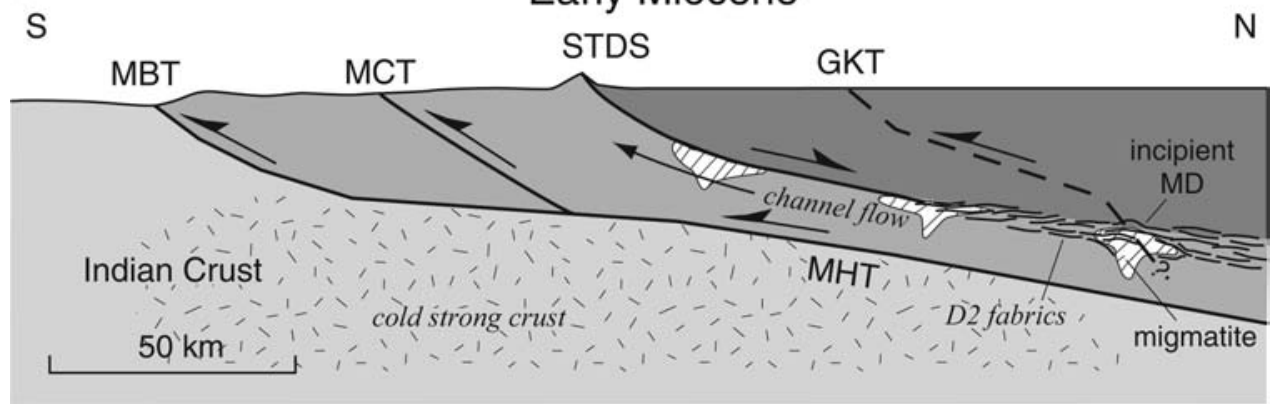

(b)

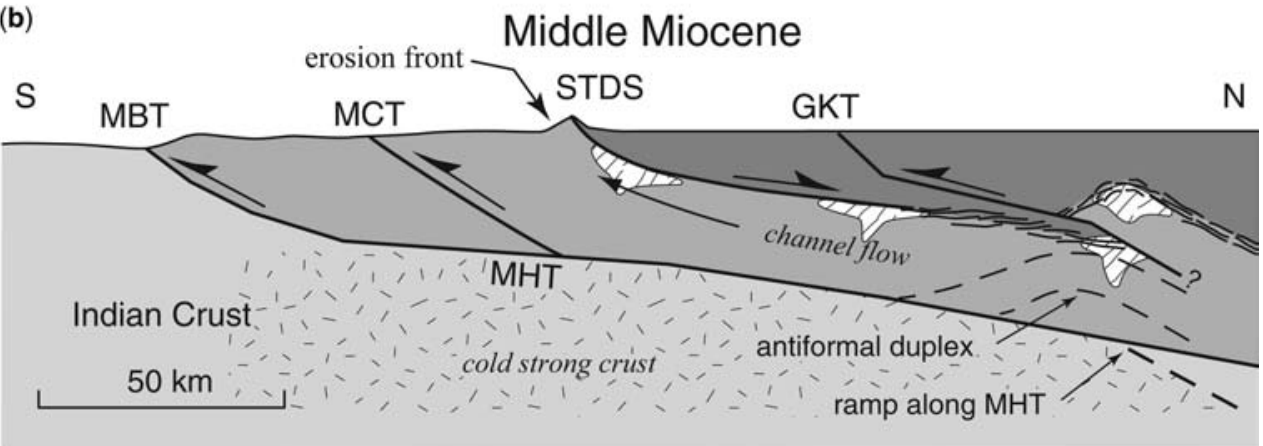

(c)

Late Miocene to Present

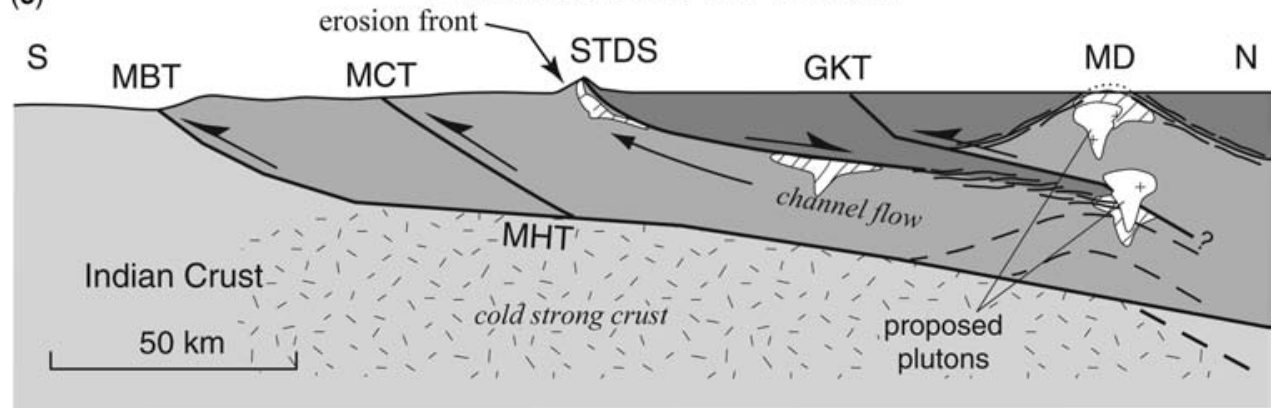

Fig. 11. Interpretative, schematic, evolutionary north-south cross-sections across the Himalaya and southern Tibet at the approximate longitude of Mabja Dome. Sections show major structures, middle-crustal migmatites, D2 horizontal stretching fabrics, cold and strong Indian crust, the GKT, and post-tectonic plutons. See text for details. Modified from Hauck et al. (1998), Wu et al. (1998), Makovsky et al. (1999), Lee et al. (2000) and Beaumont et al. (2004). GKT, Gyirong-Kangmar thrust fault system; MCT, Main Central Thrust; MD, Mabja Dome; MHT, Main Himalayan Thrust; STDS, Southern Tibetan detachment system. 
low-temperature-step potassium feldspar) and before $c .12 .5 \mathrm{Ma}$, the age of uniform cooling recorded in the low-temperature steps in potassium feldspar spectra (Fig. 7).

The structural, metamorphic and cooling histories recorded within the Mabja rocks are similar to those at Kangmar, but the intrusive histories are different (cf. Burg et al. 1984; Chen et al. 1990; Lee et al. 2000, 2004), and thus we suggest that Mabja formed by a mechanism similar to that proposed for Kangmar (Lee et al. 2000), but with some modifications (Fig. 11). As at Kangmar, normal faults were not documented that could have accommodated the middle crustal penetrative D2 vertical thinning and horizontal stretching deformation in the Mabja region. To maintain strain compatibility, we follow Lee et al.'s (2000) interpretation that these D2 deformational fabrics exposed in the core of the dome were accommodated at shallow crustal levels to the south by normal-sense (top-to-north) slip along the STDS (Fig. 11a). This interpretation implies that normal slip along the STDS must have been ongoing by at least $23 \mathrm{Ma}$, consistent with the inference that ductile shear along the STDS pre-dates $c .17 \mathrm{Ma}$ (e.g. Murphy \& Harrison 1999; Searle et al. 2003) (Fig. 11a). Moreover, our interpretation suggests that the D2 vertical thinning and horizontal stretching fabrics observed within the North Himalayan gneiss domes were accommodated by southward extrusion or channel flow of the middle crust beneath southern Tibet (e.g. Grujic et al. 1996, 2002; Searle 1999a, $b$; Beaumont et al. 2001, 2004) by at least early Miocene. The most important criterion for channel flow is low viscosity (Beaumont et al. 2001, 2004), which Beaumont et al. (2004) postulated can be achieved by a small amount of partial melt. The generation of migmatite and emplacement of a leucocratic dyke swarm, both syntectonic with the development of D2 ductile extensional deformation fabrics (Lee et al. 2004), could have provided the partial melt necessary to lower the viscosity and initiate channel flow in the middle crust of southern Tibet.

Lee et al. (2004) argued that the close spatial and temporal relations among metamorphism, partial melting, emplacement of the dyke swarm, and D2 vertical thinning and horizontal stretching in Mabja indicated that doming was, in part, driven by buoyant migmatite diapirs that were generated by adiabatic decompression during extensional collapse. We can test this high-temperature (c. $500-700^{\circ} \mathrm{C}$ ) doming mechanism by comparing the degree of fold tightness exhibited by the domed high-temperature S2 foliation and metamorphic isograds with the domed lower temperature (c. $370^{\circ} \mathrm{C}$ ) muscovite ${ }^{40} \mathrm{Ar} /{ }^{39} \mathrm{Ar}$ chrontours. If the migmatite diapir mechanism is correct, then the domal geometry defined by the $\mathrm{S} 2$ foliation and metamorphic isograds should be tighter than that defined by the ${ }^{40} \mathrm{Ar} /{ }^{39} \mathrm{Ar}$ chrontours. Both map and cross-sectional views of the $\mathrm{S} 2$ foliation, metamorphic isograds and ${ }^{40} \mathrm{Ar} /{ }^{39} \mathrm{Ar}$ chrontours show they are subparallel and do not exhibit a difference in degree of folding (Figs $8 \& 9$ ), indicating that the entire doming history in Mabja must have occurred after the rocks had cooled below $c .370^{\circ} \mathrm{C}$.

Between $c .17 \mathrm{Ma}$ and $c .15 \mathrm{Ma}$, hot Mabja rocks were captured in the hanging wall of the northdipping GKT and thrust southward up and over cold Tethyan sediments, resulting in refrigeration from below, an increase in muscovite ${ }^{40} \mathrm{Ar} /{ }^{39} \mathrm{Ar}$ ages with structural depth, rapid cooling (40-60 $\mathrm{C} /$ million years) due to refrigeration and exhumation, and 'freezing-in' middle-crustal channel flow fabrics (Fig. 11b) (cf. Lee et al. 2000). Continued movement of these rocks up and over a north-dipping ramp along the thrust fault between $c .13 \mathrm{Ma}$ and $c .12 .5 \mathrm{Ma}$ resulted in passive doming of M1 isograds, S2 foliations, and mica chrontours, and continued rapid cooling at a rate of $45-60^{\circ} \mathrm{C} /$ million years (Fig. $11 \mathrm{c}$ ). In contrast to Kangmar, muscovite ${ }^{40} \mathrm{Ar} /{ }^{39} \mathrm{Ar}$ ages observed at the deepest structural levels in Mabja decrease to $c$. 13-14 Ma, suggesting these rocks were reheated above the closure temperature for muscovite at $c$. 15-16 Ma and then rapidly cooled as a consequence of conduction and exhumation. We propose that a granite, below the present level of exposure and similar in composition and age to the two post-tectonic granites exposed, was the source of this additional heat (Fig. 11c). Symmetric cooling of the dome from c. $200^{\circ} \mathrm{C}$ to $115^{\circ} \mathrm{C}$ between $c .12 .5$ and $9.5 \mathrm{Ma}$ implies either cessation of thrust faulting and rapid exhumation $\left(45-60^{\circ} \mathrm{C} /\right.$ million years) due to erosion following thrust faulting, or continued slip along a subhorizontal portion of the GKT and erosion.

Changes in horizontal stress, shear traction at the base, rheology and/or surface height (e.g. Dahlen 1984, 1990; Davis et al. 1983; Platt 1986; England \& Molnar 1993) can explain the transition from extension to compression in an orogenic belt. We (Lee et al. 2000, 2004) speculated that increased friction along the Main Himalayan thrust (MHT) beneath the North Himalayan gneiss domes (Fig. 11) led to the change from middle crustal subhorizontal stretching deformation to contraction and formation of the GKT. Alternatively, Beaumont et al.'s (2001, 2004) thermalmechanical model of strong crust injected into and underthrusting a middle crustal channel results in the development of a north-dipping frontal ramp. The north-dipping ramp along the MHT proposed by Hauck et al. (1998) may be the leading edge of Beaumont et al.'s $(2001,2004)$ 
north-dipping frontal ramp (Fig. 11b). The cold crustal ramp provides an explanation for the development of a north-dipping ramp along the GKT proposed by Lee et al. (2000) (Fig. 11b). This hypothesis is more appealing because it does not require changes in stress, shear traction or rheology in a contractional deformation zone that is well established.

A baffling feature of the North Himalayan gneiss domes is their map geometries, which vary alongstrike from elongate north-south (Kangmar), to elongate east-west (Kampa), to asymmetric with both north-south and east-west elongate components (Mabja) (Fig. 1). To explain these variations, we propose that the shape and size of the leading edge of the cold, strong frontal ramp varies along its length. We envision that a north-south elongate, narrow footwall underthrust middle crust rocks now exposed at Kangmar, whereas an asymmetric, north-south and east-west elongate footwall underthrust Mabja. If this hypothesis is correct, then in map view the leading edge of the frontal ramp would be characterized by a sinuous pattern in which the enveloping surface strikes east-west, and lateral ramps would be developed under the east and west flanks of the domes.

There are striking parallel events among structural, metamorphic and cooling histories preserved at Mabja (Lee et al. 2004, this work) and Kangmar (Burg et al. 1984; Chen et al. 1990; Lee et al. 2000): (1) D1 deformation characterized by north-south contraction resulting in folding and thickening; (2) thermal re-equilibration of middle crustal rocks leading to peak metamorphism; (3) high-strain D2 vertical thinning and north-south horizontal stretching broadly synchronous with peak metamorphism; and (4) development of a domal geometry defined by lithologic contacts, the S2 mylonitic foliation, metamorphic isograds, and ${ }^{40} \mathrm{Ar} /{ }^{39} \mathrm{Ar}$ muscovite chrontours. Moreover, both domes lie in the hanging wall of the GKT (Fig. 1) and their domal geometries are ascribed to movement of the hangingwall of this thrust fault. Conversely, the migmatites, leucocratic dyke swarm, and post-tectonic granites of the Mabja Dome (Lee et al. 2004) were not found at Kangmar Dome (Lee et al. 2000), perhaps because Mabja exposes deeper structural levels than Kangmar. The similarities between these two domes suggest that the physical processes that produced them may be the same for other North Himalayan gneiss domes and are, therefore, of regional extent.

\section{Miocene middle crustal flow in southern Tibet}

To the south of the North Himalayan gneiss domes, the Greater Himalayan Sequence in the high
Himalaya is underlain by middle crust composed of strongly deformed high-grade metasedimentary (peak conditions of 6-7 kbar and c. $600^{\circ} \mathrm{C}$ ), orthogneissic and migmatitic rocks, and both deformed and undeformed leucogranites (e.g. Le Fort et al. 1987; Hodges et al. 1988; Hubbard 1989; Burchfiel et al. 1992; Grujic et al. 1996, 2002; Murphy \& Harrison 1999; Searle 1999a, b; Walker et al. 1999; Stephenson et al. 2000; Searle et al. 2003). Numerous $\mathrm{U}-\mathrm{Pb}$ and $\mathrm{U}-\mathrm{Th}-\mathrm{Pb}$ geochronologic ages on zircon, monazite and other uraniumbearing accessory phases, together with ${ }^{40} \mathrm{Ar} /{ }^{39} \mathrm{Ar}$ ages on micas, constrain the timing of structural, metamorphic and intrusive events recorded in these rocks. Contraction-related burial and peak Barrovian-type metamorphism (thermal reequilibration) have been bracketed between $37 \mathrm{Ma}$ and $28 \mathrm{Ma}$ (Vance \& Harris 1999; Walker et al. 1999; Simpson et al. 2000); these data also provide a minimum age for north-south contraction. $\mathrm{U}-\mathrm{Pb}$ ages, in conjunction with petrographic and trace-element partitioning observations, from the Namche migmatites in the Everest region are interpreted as indicating anatexis at $25.4-24.8 \mathrm{Ma}$ (Viskupic \& Hodges 2001). The multiple generations of both deformed and undeformed leucogranites in the high Himalaya vary in age from $31.6 \mathrm{Ma}$ to $12.5 \mathrm{Ma}$ (Noble \& Searle 1995; Hodges et al. 1996, 1998; Edwards \& Harrison 1997; Searle et al. 1997b; Wu et al. 1998; Harrison et al. 1999; Murphy \& Harrison 1999; Searle 1999a, $b$; Walker et al. 1999; Simpson et al. 2000), and their production is attributed to crustal melting either as a consequence of decompression during exhumation (e.g. Harris et al. 1993; Harris \& Massey 1994) or as a consequence of heat generated during shear stress along the Himalayan decollement (e.g. Le Fort et al. 1987; Harrison et al. 1997). Muscovite ${ }^{40} \mathrm{Ar} /{ }^{39} \mathrm{Ar}$ ages indicate that most of the high Himalaya metasediments cooled below $c .370^{\circ} \mathrm{C}$ by between $22-20 \mathrm{Ma}$ and $17-15 \mathrm{Ma}$ signifying that mylonitization ceased at this time or soon thereafter (Searle \& Rex 1989; Hodges et al. 1992; Searle et al. 1992; Walker et al. 1999; Stephenson et al. 2001). Finally, the combination of field, structural and geochronologic observations indicate that movement along the MCT and STDS shear zones, that bound the Greater Himalayan Sequence below and above, respectively, was broadly simultaneous at $c .22-13 \mathrm{Ma}$ (Hubbard \& Harrison 1989; Hodges et al. 1992, 1996; Murphy \& Harrison, 1999; Walker et al. 1999; Simpson et al. 2000; Stephenson et al. 2001; Daniel et al. 2003; Searle et al. 2003).

The structural, metamorphic, anatectic, intrusive and geochronologic histories in Mabja (Lee et al. 2004, this study), Kangmar (Burg et al. 1984; Chen et al. 1990; Lee et al. 2000), and Malashan 
(Burg et al. 1984; Aoya et al. 2006) are similar to those recorded in the Greater Himalayan Sequence, suggesting that during the late Oligocene to early Miocene high-grade middle-crustal metasedimentary and orthogneissic rocks, cross-cut by anatectic melts and leucogranites, were once continuous from beneath the high Himalaya northward beneath southern Tibet (Fig. 11a). Exposures of the Greater Himalayan Sequence have been interpreted as the leading edge of an eroding and southward-extruding tabular or wedge-shaped body of ductile middlecrustal rocks bounded above by the STDS and below by the MCT (e.g. Grujic et al. 1996, 2002; Nelson et al. 1996; Searle 1999a, b; Beaumont et al. 2001, 2004; Hodges et al. 2001; Vannay \& Grasemann 2001; Searle et al. 2003). Beaumont et al.'s (2001, 2004) thermal-mechanical models predict that channel flow within the middle crust will develop in the Himalayan orogen if low-viscosity partial melt is present in the middle crust, differential lithostatic pressure is established across the orogen, and surface denudation occurs along the southern flank of the Himalaya. We suggest that the hot and weak middle crustal rocks now exposed in the core of the North Himalayan gneiss domes represent the interior of such a middle-crustal channel. Presentday exposures of mid-crustal rocks in the gneiss dome core signify that a piece of the middle-crustal channel was excised in southern Tibet. Our interpretation that exhumation of these high-grade rocks was related to their movement up and over a strong, crustal frontal ramp (Beaumont et al. 2001, 2004) and into the hanging wall of the GKT during the middle Miocene provides a mechanism by which this piece of the flowing middle crustal channel was cut out (Fig. 11b). New and previously published geochronologic data indicate that ductile flow was synchronous in the core of Mabja Dome and in the high Himalaya, implying that the middle crust throughout southern Tibet and the high Himalaya was, in general, flowing southward by early Miocene times.

Studies along the southern flank of the Himalaya have concluded that the deformation field of the channel flow tunnel or extruding wedge can be described as shear along the MCT and STDS (e.g. Hodges et al. 1993), simple shear distributed throughout the wedge (Grujic et al. 1996), or general shear flow concentrated along the boundaries of the wedge with pure shear in the centre (Grasemann et al. 1999). Although sparse, kinematic data from the North Himalayan gneiss domes (e.g. Chen et al. 1990; Lee et al. 2000, 2004; Aoya et al. 2005, 2006) imply that these middle-crustal rocks were probably deformed along the upper part of the deforming wedge or channel. Detailed quantitative kinematic studies may resolve where in the channel these rocks were deformed.
Geophysical observations, including short-wavelength gravity anomalies (Jin et al. 1994), and the coincidence of high electrical conductivity, middle-crustal low velocities, and reflection bright spots (Chen et al. 1996; Makovsky et al. 1996; Nelson et al. 1996; Alsdorf \& Nelson 1999), suggest that the middle crust beneath southern Tibet is currently hot, partially molten, and weak. The migmatites and leucogranites in the high Himalaya and in the North Himalayan gneiss domes are exposures of the once hot and weak Oligocene-Miocene middle crust (Nelson et al. 1996; Searle et al. 2003; Lee et al. 2004).

\section{Conclusions}

New isotopic ages from the Mabja Dome reveal a late Oligocene to early Miocene history of ductile vertical thinning and horizontal stretching, peak metamorphism, migmatization and emplacement of a leucocratic dyke swarm, early to middle Miocene south-vergent thrust faulting resulting in doming, and post-tectonic emplacement of middle Miocene two-mica granites. Our $23.1 \pm 0.8 \mathrm{Ma}$ $\mathrm{U}-\mathrm{Pb}$ zircon age from a deformed leucocratic dyke are the first to constrain the timing of ductile extension, metamorphism and migmatization within the North Himalayan gneiss domes. Mica ${ }^{40} \mathrm{Ar} /{ }^{39} \mathrm{Ar}$ and apatite fission track cooling ages indicate rapid cooling and doming during the middle Miocene. Rapid cooling is attributed to both refrigeration from below and exhumation. The domal geometry observed at Mabja is solely ascribed to tectonically driven south-vergent thrust faulting. The similar structural, metamorphic, intrusive and timing histories at Mabja Dome and in the Greater Himalayan sequence imply that during late Oligocene to early Miocene times, high-grade metasedimentary rocks and orthogneissic rocks, intruded by migmatites and leucogranites, were continuous in the middle crust from beneath the High Himalaya northward to beneath southern Tibet. These middle crustal rocks have been interpreted as a southward-flowing middle crustal channel, with the Greater Himalayan Sequence defining the eroding and extruding leading edge. In southern Tibet, a slice of the southward-flowing middle crustal channel was excised by southvergent thrust faulting during the middle Miocene, explaining the present-day exposures of highgrade rocks in the cores of the North Himalayan gneiss domes. Excisement via thrust faulting was broadly simultaneous with normal slip along the STDS and reverse slip along the MCT.

M. Harrison, R. Law and S. Noble provided valuable comments that improved this manuscript. Funding for this project was provided by National Science Foundation 
grant EAR-9526861, National Science Foundation Grant of China 49473171, and Central Washington University. We are grateful to J. Wooden for his help with data collection and analysis at the Stanford-US Geological Survey SHRIMP-RG facility.

\section{References}

Alsdorf, D. \& Nelson, D. 1999. Tibetan satellite magnetic low: evidence for widespread melt in the Tibetan crust? Geology, 27, 943-946.

Aoya, M., Wallis, S. R., Terada, K., Lee, J., Kawakami, T., Wang, Y. \& Heizler, M. 2005. North-south extension in the Tibetan crust triggered by granite emplacement. Geology, 33, $853-856$.

Aoya, M., Wallis, S. R., Kawakami, T., Lee, J., WAng, Y. \& MAEdA, H. 2006. The Malashan gneiss dome in south Tibet: comparative study with the Kangmar Dome with special reference to kinematics of deformation and origin of associated granites. In: LAW, R. D., SEARle, M. P. \& Godin, L. (eds) Channel Flow, Ductile Extrusion and Exhumation in Continental Collision Zones. Geological Society, London, Special Publications, 268, 471-495.

Armijo, R., Tapponnier, P., Mercier, J. \& Han, T. 1986. Quaternary extension in southern Tibet: Field observations and tectonic implications. Journal of Geophysical Research, 91, 13,803-13,872.

Beaumont, C., Jamieson, R. A., Nguyen, M. H. \& LEE, B. 2001. Himalayan tectonics explained by extrusion of a low-viscosity crustal channel coupled to focused surface denudation. Nature, 414, 738-742.

Beaumont, C., Jamieson, R. A., Nguyen, M. H. \& SERGEI, M. 2004. Crustal channel flows: 1. Numerical models with application to the tectonics of the Himalayan-Tibetan orogen. Journal of Geophysical Research, 109. DOI: 10.1029/2003JB002809.

Black, L. P., Kamo, S. L., Williams, I. S., Mundil, R., Davis, D. W., Korsch, R. J. \& Foudoulis, C. 2003. The application of SHRIMP to Phanerozoic geochronology; a critical appraisal of four zircon standards. Chemical Geology, 200, $171-188$.

Burchfiel, B. C., Zhiliang, C., Hodges, K. V., Yuping, L., Royden, L. H., Changrong, D. \& JiEnE, X. 1992. The south Tibetan detachment system, Himalayan orogen: Extension contemporaneous with and parallel to shortening in a collisional mountain belt. Geological Society of America Special Paper 269.

Burg, J. P. \& Chen, J. M. 1984. Tectonics and structural zonation of southern Tibet, China. Nature, 311, 219-223.

Burg, J. P., Guiraud, M., Chen, G. M. \& Li, G. C. 1984. Himalayan metamorphism and deformations in the north Himalayan Belt (southern Tibet, China). Earth and Planetary Science Letters, 69, 391-400.

Chen, Z., Liu, Y., Hodges, K. V., Burchfiel, B. C., Royden, L. H. \& DenG, C. 1990. The Kangmar
Dome: A metamorphic core complex in southern Xizang (Tibet). Science, 250, 1552-1556.

Chen, L., Booker, J. R., Jones, A. G., Nong, W. Unsworth, M. J., Wenbo, W. \& Handong, T. 1996. Electrically conductive crust in southern Tibet from INDEPTH magnetotelluric surveying. Science, 274, 1694-1696.

Cherniak, D. J. \& Watson, E. B. 2003. Diffusion in zircon. In: Hanchar, J. M. \& Hoskin, P. W. O. (eds) Zircon. Mineralogical Society of America, Washington, Reviews in Mineralogy and Geochemistry, 53, 113-143.

Dahlen, F. A. 1984. Noncohesive critical coulomb wedges: An exact solution. Journal of Geophysical Research, 89, 10,125-10,133.

DAHLEN, F. A. 1990. Critical taper model of fold-andthrust belts and accretionary wedges. Annual Reviews of Earth and Planetary Sciences, 18, 55-99.

Daniel, C. G., Hollister, L. S., Parrish, R. R. \& GrujIC, D. 2003. Exhumation of the Main Central Thrust from lower crustal depths, eastern Bhutan Himalaya. Journal of Metamorphic Geology, 21, 317-334.

Davis, D., Suppe, J. \& Dahlen, F. A. 1983. Mechanics of fold-and-thrust belts and accretionary wedges. Journal of Geophysical Research, 88, $1153-1172$.

Edwards, M. A. \& Harrison, T. M. 1997. When did the roof collapse? Late Miocene N-S extension in the high Himalaya revealed by $\mathrm{Th}-\mathrm{Pb}$ dating of the Khula Kangri granite. Geology, 25, 543-546.

England, P. \& Molnar, P. 1993. Cause and effect among thrust faulting and normal faulting, anatectic melting and exhumation in the Himalaya, In: Treloar, P. J. \& Searle, M. P. (eds) Himalayan Tectonics. Geological Society, London, Special Publications, 74, 401-411.

Gaetani, M. \& Garzanti, E. 1991. Multicycle history of the northern India continental margin (Northwestern Himalaya). American Association of Petroleum Geologists Bulletin, 75, 1427-1446.

Galbraith, R. F. 1981. On statistical methods of fission track counts. Mathematical Geology, 13, 471-478.

Galbraith, R. F. \& Laslett, G. M. 1993. Statistical models for mixed fission track ages. Nuclear Tracks Radiation Measurements, 21, 459-470.

GANSSER, A. 1964. Geology of the Himalayas. Wiley-Interscience, London.

Godin, L., Brown, R. L., HANMER, S. \& PARrish, R. 1999. Back folds in the core of the Himalayan orogen: An alternative interpretation. Geology, 27, $151-154$.

Grasemann, B., Fritz, H. \& Vannay, J.-C. 1999. Quantitative kinematic flow analysis from the Main Central Thrust zone (NW-Himalaya, India): implications fro a decelerating strain path and the extrusion of orogenic wedges. Journal of Structural Geology, 21, 837-853.

GreEN, R. 1981. A new look at statistics in fission track dating. Nuclear Tracks Radiation Measurements, 5, 77-86. 
Grove, M. \& Harrison, T. M. $1996 .{ }^{40}$ Ar*diffusion in Fe-rich biotite. American Mineralogist, 81, 940-951.

Grujic, D., Casey, M., Davidson, C., Hollister, L. S., Kundig, R., Pavlis, T. \& Schmid, S. 1996. Ductile extrusion of the High Himalayan Crystalline in Bhutan: evidence from quartz microfabrics. Tectonophysics, 260, 21-43.

Grujic, D., Hollister, L. S. \& Parrish, R. R. 2002. Himalayan metamorphic sequence as an orogenic channel: insight from Bhutan. Earth and Planetary Sciences, 198, 177-191.

Harris, N. \& MASSEY, J. A. 1994. Decompression and anatexis of Himalayan metapelites. Tectonics, 13, $1537-1546$.

Harris, N. B. W., Inger, S. \& Massey, J. A. 1993. The role of fluids in the formation of High Himalayan leucogranites. In: Treloar, P. J. \& SeArle, M. P. (eds) Himalayan Tectonics. Geological Society, London, Special Publications, 74, 391-400.

HARRISON, T. M. 1981. Diffusion of ${ }^{40} \mathrm{Ar}$ in hornblende. Contributions to Mineralogy and Petrology, 78, 324-331.

Harrison, T. M., Ducan, I. \& McDougall, I. 1985. Diffusion of ${ }^{40} \mathrm{Ar}$ in biotite-Temperature, pressure and compositional effects. Geochimica et Cosmochimica Acta, 49, 2461-2468.

Harrison, T. M., Copeland, P., Kidd, W. S. F. \& LOVERA, O. M. 1995. Activation of the Nyainqentanghla shear zone: Implications for uplift of the southern Tibetan Plateau. Tectonics, 14, 658-676.

Harrison, T. M., Lovera, O. M. \& Grove, M. 1997. New insights into the origin of two contrasting Himalayan granite belts. Geology, 25, 899-902.

Harrison, T. M., Grove, M., McKeegan, K. D., CoAth, C. D., Lovera, O. \& Le Fort, P. 1999. Origin and episodic emplacement of the Manaslu Intrusive Complex, Central Himalaya. Journal of Petrology, 40, 3-19.

Hauck, M. L., Nelson, K. D., Brown, L. D., Zhao, W. \& Ross, A. R. 1998. Crustal structure of the Himalayan orogen at $\sim 90^{\circ}$ east longitude from Project INDEPTH deep reflection profiles. Tectonics, 17, 481-500.

Hodges, K. V., Hubbard, M. S. \& Silverberg, D. S. 1988. Metamorphic constraints on the thermal evolution of the central Himalayan orogen. Philosophical Transactions of the Royal Society, London, A326, 257-280.

Hodges, K. V., Parrish, R. R., Hoish, T. B., LuX, D. R., Burchfiel, B. C., Royden, L. H. \& Chen, Z. 1992. Simultaneous Miocene extension and shortening in the Himalaya orogen. Science, 258, 1466-1469.

Hodges, K. V., Burchfiel, B. C., Royden, L. H., Chen, Z. \& LiU, Y. 1993. The metamorphic signature of contemporaneous extension and shortening in the central Himalayan orogen: data from Nyalam transect, southern Tibet. Journal of Metamorphic Geology, 11, 721-737.

Hodges, K. V., Parrish, R. R. \& Searle, M. P. 1996. Tectonic evolution of the central Annapurna range, Nepalese Himalayas. Tectonics, 15, 1264-1291.
Hodges, K. V., Bowring, S. A., DAvidek, K., Hawkins, D. \& Krol, M. 1998. Evidence for rapid displacement on Himalayan normal faults and the importance of tectonic denudation in the evolution of mountain ranges. Geology, 26, $483-486$.

Hodges, K. V., Hurtado, J. M. \& Whipple, K. X. 2001. Southward extrusion of Tibetan crust and its effect on Himalayan tectonics. Tectonics, 20, 799-809.

HubBARD, M. S. 1989. Thermobarometric constraints on the thermal history of the Main Central Thrust zone and Tibetan Slab, eastern Nepal Himalaya. Journal of Metamorphic Geology, 7, 127-134.

HubBard, M. S. \& HARRison, T. M. $1989 .{ }^{40} \mathrm{Ar} /{ }^{39} \mathrm{Ar}$ age constraints on deformation and metamorphism in the Main Central Thrust zone and Tibetan slab, eastern Nepal Himalaya. Tectonics, 8, 865-880.

Hurford, A. J. \& GREen, F. 1983. The Zeta age calibration of fission-track dating. Isotope Geoscience, 1, 285-317.

Jin, Y., McNutT, M. K. \& Zhu, Y. 1994. Evidence from gravity and topography data for folding of Tibet. Nature, 371, 669-674.

LE FORT, P. 1975. Himalayas: The collided range. Present knowledge of the continental arc. American Journal of Science, 275-A, 1-44.

LE FORT, P. 1986. Metamorphism and magmatism during the Himalayan. In: CowARD, M. P. \& ReIs, A. C. (eds) Collision Tectonics. Geological Society, London, Special Publications, 19, 159-172.

Le Fort, P., Cuney, M., Deniel, C., FranceLanord, C., Sheppard, S. M. F., Upreti, B. N. \& VIDAL, P. 1987. Crustal generation of the Himalayan leucogranites. Tectonophysics, 134, 39-57.

LEE, J. 1995. Rapid uplift and rotation of mylonites: Insights from potassium feldspar ${ }^{40} \mathrm{Ar} /{ }^{39} \mathrm{Ar}$ thermochronology, northern Snake Range, Nevada. Tectonics, 14, 54-77.

LeE, J., Dinklage, W. S., Hacker, B. R. ET AL. 2000. Evolution of the Kangmar Dome, southern Tibet: Structural, petrologic, and thermochronologic constraints. Tectonics, 19, 872-896.

LeE, J., HACKer, B. R. \& WANG, Y. 2004. Evolution of North Himalayan Gneiss Domes: Structural and metamorphic studies in Mabja Dome, southern Tibet. Journal of Structural Geology, 26, 22972316.

Lister, G. S. \& BALDwin, S. L. 1996. Modelling the effect of arbitrary P-T-t histories on argon diffusion in minerals using the MacArgon program for the Apple Macintosh. Tectonophysics, 253, 83-109.

LUDWIG, K. R. 1999. Isoplot/EX version 2.10: a geochronological toolkit for Microsoft Excel. Berkeley Geochronology Center, Special Publication, 1.

LudwiG, K. R. 2001. Squid version 1.02: A users manual. Berkeley Geochronology Center, Special Publication, 2.

McClelland, W. C. \& Mattinson, J. M. 1996. Resolving high precision ages from Tertiary plutons with complex zircon systematics. Geochimica et Cosmochimica Acta, 60, 3955-3965. 
Makovsky, Y., Klemperer, S. L., Liyan, H. \& DEYUAN, L. 1996. Structural elements of the southern Tethyan Himalaya crust from wideangle seismic data. Tectonics, 15, 997-1005.

Maluski, H., Matte, P. \& Brunel, M. 1988. Argon 39-Argon 40 dating of metamorphic and plutonic events in the North and High Himalayas belts (southern Tibet-China). Tectonics, 7, 299-326.

Mancktelow, N. S. \& Grasemann, B. 1997. Timedependent effects of heat advection and topography on cooling histories during erosion. Tectonophysics, 270, 167-195.

Mattinson, J. M. 1987. U-Pb ages of zircons: a basic examination of error propagation. Chemical Geology, 66, 151-162.

Mercier, J.-L., Armijo, R., TApponnier, P., CAREyGailhardis, E. \& Lin, H. T. 1987. Change from late Tertiary compression to Quaternary extension in southern Tibet during the India-Asia collision. Tectonics, 6, 275-304.

Molnar, P. \& TApponnier, P. 1975. Effects of a continental collision. Science, 189, 419-426.

Moore, M. A. \& England, P. C. 2001. On the inference of denudation rates from cooling ages of minerals. Earth and Planetary Sciences, $\mathbf{1 8 5}$, 265-284.

Murphy, M. A. \& Harrison, T. M. 1999. Relationship between leucogranites and the Qomolangma detachment in the Rongbuk Valley, south Tibet. Geology, 27, 831-834.

NAESER, C. W. 1976. Fission track dating. US Geological Survey Open File Report, 76-190.

NAESER, C. W. 1979. Fission-track dating and geologic annealing of fission tracks. In: JAEGER, E. \& HUNZIKER, J. C. (eds) Lectures in Isotope Geology. Springer-Verlag, New York, 154-169.

Nelson, K. D., Zhao W., Brown, L. D. ET AL. 1996. Partially molten middle crust beneath southern Tibet: Synthesis of project INDEPTH results. Science, 274, 1684-1688.

Noble, S. R. \& Searle, M. P. 1995. Age of crustal melting and leucogranite formation from $\mathrm{U}-\mathrm{Pb}$ zircon and monazite dating in the western Himalaya, Zanskar, India. Geology, 23, 1135-1138.

Platt, J. P. 1986. Dynamics of orogenic wedges and the uplift of high-pressure metamorphic rocks. Geological Society of America Bulletin, 97, $1037-1053$

Quidelleur, X., Grove, M., Lovera, O. M., Harrison, T. M., Yin, A. \& RYerson, F. J. 1997. The thermal evolution and slip history of the Renbu Zedong thrust, southeastern Tibet. Journal of Geophysical Research, 102, 2659-2679.

Ratschbacher, L., Frisch, W., Liu, G. \& Chen, C. 1994. Distributed deformation in southern and western Tibet during and after the India-Asia collision. Journal of Geophysical Research, 99, $19,917-19,945$.

Scharer, U., Xu, R. \& Allegre, C. 1986. U-(Th)-Pb systematics and ages of Himalayan leucogranites, south Tibet. Earth and Planetary Science Letters, 77, 35-48.

SEARLE, M. P. 1983. Stratigraphy, structure and evolution of the Tibetan-Tethys zone in Zanskar and the
Indus suture zone in the Ladakh Himalaya. Transactions of the Royal Society of Edinburgh, 73, 205-219.

Searle, M. P. 1999a. Extensional and compressional faults in the Everest-Lhotse massif, Khumbu Himalaya, Nepal. Journal of the Geological Society, London, 156, 227-240.

Searle, M. P. 1999b. Emplacement of Himalayan leucogranites by magma injection along giant sill complexes: examples from the Cho Oyu, Gyachung Kang and Everest leucogranites (Nepal Himalaya). Journal of Asian Earth Sciences, 17, $773-783$.

Searle, M. P. \& ReX, D. C. 1989. Thermal model for the Zanskar Himalaya. Journal of Metamorphic Geology, 7, 127-134.

Searle, M. P., Waters, D. J., Rex, D. C. \& Wilson, R. N. 1992. Pressure, temperature and time constraints on Himalayan metamorphism from eastern Kashmir and western Zanskar. Journal of the Geological Society, London, 149, 753-773.

Searle, M., Corfield, R. I., Stephenson, B. \& MCCARRON, J. 1997a. Structure of the north Indian continental margin in the Ladakh-Zanskar Himalayas: Implications for the timing of obduction of the Spontang ophiolite, India-Asia collision and deformational events in the Himalaya. Geological Magazine, 134, 297-316.

Searle, M. P., Parrish, R. R., Hodges, K. V., Hurford, A., Ayres, M. W. \& Whitehouse, M. J. 1997b. Shisha Pangma leucogranite, south Tibetan Himalaya: Field relations, geochemistry, age, origin, and emplacement. Journal of Geology, 105, 295-317.

Searle, M. P., Simpson, R. L., Law, R. D., Parrish, R. R. \& WATERS, D. J. 2003. The structural geometry, metamorphic and magmatic evolution of the Everest massif, High Himalaya of Nepal-South Tibet. Journal of the Geological Society, London, 160, 345-366.

Simpson, R. L., Parrish, R. R., Searle, M. P. \& WATERS, D. J. 2000. Two episodes of monazite crystallization during metamorphism and crustal melting in the Everest region of the Nepalese Himalaya. Geology, 28, 403-406.

Stacey J. S. \& KRAMERS, J. D. 1975. Approximation of terrestrial lead isotope evolution by a two-stage model. Earth and Planetary Science Letters, 26. 207-221.

Stephenson, B. J., Waters, D. J. \& Searle, M. P. 2000. Inverted metamorphism and the Main Central Thrust: field relations and thermobarometric constraints from the Kishtwar Window, NW Indian Himalaya. Journal of Metamorphic Geology, 18, 571-590.

Stephenson, B. J., Searle, M. P., Waters, D. J. \& Rex, D. C. 2001. Structure of the Main Central Thrust zone and extrusion of the High Himalayan deep crustal wedge, Kishtwar-Zanskar Himalaya. Journal of the Geological Society, London, 158, 637-652.

Tapponnier, P., Peltzer, G., Le Dain, A. Y., Armijo, R. \& CobBold, P. 1982. Propagating extrusion tectonics in Asia: New insights from 
simple experiments from plasticine. Geology, 10, 611-615.

VANCE, D. \& HARRIS, N. 1999. The timing of prograde metamorphism in the Zanskar Himalaya. Geology, 27, 395-398.

VANNAY, J.-C. \& Grasemann, B. 2001. Himalayan inverted metamorphism and syn-convergence extension as a consequence of a general shear extrusion. Geological Magazine, 138, 253-276.

Viskupic, K. \& Hodges, K. V. 2001. Monazitexenotime thermochronometry: methodology and an example from the Nepalese Himalaya. Contributions to Mineralogy and Petrology, 141, $233-247$.

Walker, J. D., Martin, M. W., Bowring, S. A., Searle, M. P., Waters, D. J. \& Hodges, K. V. 1999. Metamorphism, melting, and extension: Age constraints from the High Himalayan slab, S. E. ZANSKAR and N. W. LAHOUL. Journal of Geology, 107, 473-495.

Williams, I. S. 1998. U-Pb by ion microprobe. In: McKibben, M. A., Shanks, W. C. \& Ridley, W. I. (eds) Applications of Microanalytical
Techniques to Understanding Mineralizing Processes. Society of Economic Geologists, Reviews in Economic Geology, 7, 1-35.

Wu, C., Nelson, K. D., Wortman, G. et al. 1998. Yadong cross structure and South Tibetan Detachment in the east central Himalaya $\left(89^{\circ}-90^{\circ} \mathrm{E}\right)$. Tectonics, 17, 28-45.

Yin, A., Harrison, T. M., Ryerson, F. J., Chen, W., KIDD, W. S. F. \& Copeland, P. 1994. Tertiary structural evolution of the Gangdese thrust system, southeastern Tibet. Journal of Geophysical Research, 99, 18,175-18,201.

Yin, A., Harrison, T. M., Murphy, M. A. ET AL. 1999. Tertiary deformational history of southeastern and southwestern Tibet during Indo-Asian collision. Geological Society of America Bulletin, 111, 1644-1664.

Zhang, H., Harris, N., Parrish, R., Kelley, S., Zhang, L., Rogers, N., Argles, T. \& King, J. 2004. Causes and consequences of protracted melting of the mid-crust exposed in the North Himalayan antiform. Earth and Planetary Science Letters, 228, 199-212. 
